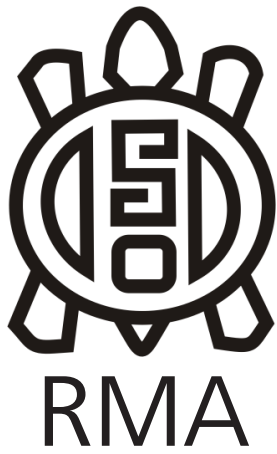

Arqueología

\title{
Biogeografía Humana y Tendencia Demográfica en el Monte Nordpatagónico. Una aproximación arqueológica desde El Corcovo (SE de Mendoza)
}

\author{
Human Biogeography and Demographic Trend in Northpatagonic Monte: \\ An Archaeological Approach from "El Corcovo" (SE of Mendoza) \\ Adolfo F. Gil*, Nuria Sugrañes**, Agustín Acevedo***, \\ Gustavo Neme****, Laura Salgán*****, Miguel Giardina******, \\ Hugo Tucker $* * * * * *$, Danae Fiore $* * * * * * *$, Viviana P. Seitz ${ }^{* * * * * * * * *}$,

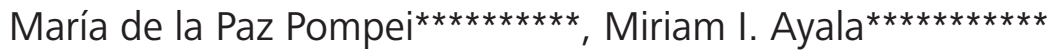

\begin{abstract}
*Instituto de Evolución, Ecología Histórica y Ambiente (CONICET-UTN FRSR); UNCuyo. agil@mendoza-conicet.gob.ar **Instituto de Evolución, Ecología Histórica y Ambiente (CONICET-UTN FRSR). nuria30@gmail.com ***CONICET, Asociación de Investigaciones Antropológicas. agustinacevedo2009@gmail.com ****Instituto de Evolución, Ecología Histórica y Ambiente (CONICET-UTN FRSR). gneme@mendoza-conicet.gob.ar *****Instituto de Evolución, Ecología Histórica y Ambiente (CONICET-UTN FRSR)-International Center of Earth Science (UNCUyo-CNEA) mlaurasalgan@gmail.com ******Instituto de Evolución, Ecología Histórica y Ambiente (CONICET-UTN FRSR). mgiardina@mendoza-conicet.gob.ar $* * * * * *$ CRIDC-Museo Regional Malargue (Mendoza. Argentina). tuckerhugo@gmail.com $* * * * * * *$ CONICET, UBA, Asociación de Investigaciones Antropológicas. danae_fiore@yahoo.es ********Instituto Argentino de Investigaciones de Zonas Áridas. vseitz@mendoza-conicet.gob.ar $* * * * * * * *$ Instituto de Evolución, Ecología Histórica y Ambiente (CONICET-UTN FRSR). mpompei@mendoza-conicet.gob.ar $* * * * * * * * * *$ Museo de Historia Natural de San Rafael. miruayalalopez@gmail.com
\end{abstract}

\begin{abstract}
Resumen
El presente trabajo aporta al conocimiento de la biogeografía humana en ambientes áridos y las fases de poblamiento en relación con las trayectorias demográficas humanas. En ese marco, se presenta nueva información arqueológica del área El Corcovo, emplazada en el Monte surmendocino/nordpatagónico. Se detallan los trabajos de campo que contemplaron transectas, excavación arqueológica de un sitio y el relevamiento de arte rupestre de otros tres. Los resultados señalan el inicio de las ocupaciones en torno a 1900 años 14C AP y un cambio en el patrón de movilidad en torno a 600 años 14C AP. Ambas situaciones son consistentes con las estimaciones cronológicas relativas existentes para el arte rupestre del área. Con base en un modelo demográfico regional del Monte, los resultados encuentran dos pulsos de incremento en la señal humana que se corresponden cronológicamente con la fecha inicial de ocupación estimada para el área y con la fecha obtenida para el cambio propuesto. Al asociarse estos pulsos con disminuciones significativas en el sector fitogeográfico de Patagonia se postulan explicaciones alternativas sobre la dinámica poblacional.
\end{abstract}

Palabras clave: Arqueología; Mendoza; Holoceno tardío; Monte; Nordpatagonia; Biogeografía humana; Arte rupestre; Cazadores-recolectores; Movilidad.

\begin{abstract}
The present paper attempt to improve the knowledge of human biogeography in arid environments and, understand the phases of settlement in relation to human demographic trajectories. In this framework, new archaeological information of the El Corcovo area is presented, located in Monte Surmendocino / Nordpatagónico. The field-works that contemplated transects, archaeological excavation of a site and the surveying of rock art of three others are detailed. The results indicate the beginning of occupations around 1900 years $14 \mathrm{C} \mathrm{AP}$ and a change in the pattern of mobility around 600 years 14C AP. Both situations are consistent with the existing relative chronological estimates for the rock art of the area. Based on a regional demographic model for Monte area, the results show two pulses of increase in the human signal that correspond chronologically with the initial date of occupation estimated for the area and with the date obtained for the proposed change. By associating these pulses with significant falls in the phyto-geographic sector of Patagonia, alternative explanations on population dynamics are postulated.
\end{abstract}

Keywords: Archaeology; Mendoza; Late Holocene; Monte; Northpatagonia; Human biogeography; rock art; huntergatherer; mobility. 
Las estrategias generadas por los grupos humanos ante situaciones socio-ambientales críticas, como las que plantean los ambientes áridos, pueden ser comprendidas mediante estudios de escalas temporales largas (Yellen 1977). Así se obtienen patrones no observables en escala histórica o etnográfica. Desde ese enfoque, un primer acercamiento biogeográfico se apoya en la definición de los procesos de exploración, colonización y ocupación efectiva regionales (Borrero 1989). Complementando esa perspectiva, las tendencias demográficas informan, en esa escala, sobre procesos de variación en relación a los recursos y cambios en la organización del sistema humano-ambiental. Es una variable central en la explicación de los procesos de poblamiento y los cambios organizacionales. Estudios previos, influidos por modelos generados en la ecología, han mostrado que para entender el poblamiento de regiones con estas características, no sólo es importante la baja productividad que caracteriza estos ambientes sino también el grado de variabilidad intra e interanual en los mismos (Gould 1991; Jochim 1981; Yellen 1977). Junto a esta idea, se resalta que el concepto de ambiente árido (o "desierto") implica un amplio rango de estructuras ambientales, aunque mantienen en común el déficit hídrico entre las precipitaciones y la evapotranspiración. Estas diferencias, que quedan desdibujadas bajo el concepto de "desierto", podrían implicar diferentes formas que las poblaciones humanas se incorporan y evolucionan en un sistema de este tipo. El Noroeste patagónico, especialmente en el Sur de Mendoza, permite considerar algunas de estas variaciones dentro de los desiertos; esto es claramente demostrado por la definición, basado en unidades fitogeográficas de desiertos contiguos que la conforman: Altoandino, Patagonia y Monte. Aunque en la última década los trabajos de investigación se han intensificado (Aguerre 2002; Gordón et al. 2017, 2018; Neme y Gil 2005), aún el conocimiento sobre el pasado humano prehistórico en el Monte $y$, consecuentemente, de su registro arqueológico, es escaso y proviene, principalmente, de sectores por fuera del Monte Nordpatagónico. Estos trabajos señalaron la débil y discontinua ocupación del Monte registrándose, hacia fines del Holoceno tardío, una ocupación efectiva del mismo (Neme y Gil 2008). Pero tal patrón podría ser consecuencia de una baja intensidad en los trabajos de campo y, por ello, la necesidad de mejorar la base de información disponible. Intentando cambiar este panorama, este artículo pretende aportar recientes resultados del sector sureste de Mendoza, específicamente del área El Corcovo (figura $1)$, del cual se carecen estudios previos.

Tendencias en el registro arqueológico del Noroeste patagónico como marco para el estudio de El Corcovo

Los datos disponibles para el noroeste patagónico, delimitado aquí por el Centro Norte de Neuquén, Sur de Mendoza y Oeste de La Pampa, señalan el inicio de la presencia humana desde unos 10.100 a 9.000 años 14C AP (Barberena 2015; Cordero et al. 2002; Neme y Gil 2012) o un poco antes según la modelación con datos biológicos y radiocarbónicos (Pérez et al. 2016). Las evidencias arqueológicas de estas primeras ocupaciones no están claramente asociadas a fauna extinta, situación contrastante con otros sectores de Patagonia y Pampa (Borrero 2009; Martínez et al. 2016; Prates et al. 2013; Steele y Politis 2009). La tecnología lítica señala un uso principalmente de rocas locales (Neme 2007; Neme et al. 2011; Rughini 2016). En el sector Monte Nordpatagónico, los datos más tempranos de ocupación se remontan al Holoceno temprano (Casa de Piedra-1, Gradin 1984; Cueva Delerma, Gil 2006) y, claramente, ya en la segunda mitad del Holoceno tardío. Iniciado el Holoceno medio, posterior a los 7.000 años AP, luego de un posible incremento en la demografía de estas poblaciones, se registra una caída y casi desaparición de la señal arqueológica (Gil 2006; Gil et al. 2005; Neme y Gil 2009) en ambos desiertos. Esta situación ha impulsado un debate sobre este cambio en la secuencia arqueológica y sus posibles causas (Barberena et al. 2015; Durán y Mikkan 2009; Durán et al. 2016; García 2010; Gil et al. 2005; Méndez et al. 2014; Neme y Gil 2009, 2010). En torno a los 5000 años $A P$ y, principalmente post 4000 años $A P$, los restos de ocupaciones humanas inician nuevamente su registro en el sector patagónico (Barberena et al. 2009; Neme y Gil 2012; Neme et al. 2005). En el Monte, esta señal arqueológica se retoma con intensidad luego de los 2000 años AP (Barberena et al. 2015; Neme et al. 2005).

Se ha propuesto que ocurrieron otros cambios importantes alrededor de 2000 años AP, principalmente como consecuencia de un incremento en la densidad demográfica (Gil et al. 2014; Neme 2007). Hacia el Norte del área, en el Monte y, principalmente en los alrededores del cerro El Nevado y el valle del río Atuel, se registran plantas domésticas (maíz, zapallo, poroto y quínoa; Gil et al. 2014), mientras que en el sector patagónico, este registro es muy efímero y básicamente restringido a Lagenaria sp. (Gil et al. 2014). Durante esta segunda parte del Holoceno tardío, se producen innovaciones en la organización tecnológica incluyendo la incorporación de cerámica (Sugrañes y Franchetti 2012) y cambios en la organización de la tecnología lítica indicada, entre otras cosas, por incorporación de nuevas armas de caza e incremento en el uso de materias primas no locales (Neme 2002; Pompei 2018; Salgán 2013).

El Oeste de nordpatagonia es un área con significativa variabilidad ambiental plasmada en los tres desiertos que ocupan la mayor parte del área: Altoandina, Patagonia y Monte. Por ello, se necesita discutir hasta qué punto esta heterogeneidad ambiental fue significativa en la estructuración de la estrategias humanas y en la evolución del sistema. Estudios previos muestran una débil y discontinua señal humana hasta unos 2000 años 
AP. En los sectores asociados a valles fluviales significativos, como los ríos Atuel, Diamante y Colorado este patrón sería distinto (Neme et al. 2005). Junto a esto, la estructura arqueológica ha señalado que la ocupación efectiva sería en el Holoceno tardío, particularmente en los últimos 2000 y 1000 años (Gil 2006, Neme et al. 2005). Los pocos sitios estudiados han sido la base para sostener esta tendencia. Por ello, este trabajo busca aportar nuevos datos del Monte Nordpatagónico, centrados en el área de El Corcovo (Sureste de Mendoza). En el año 2005, el primer trabajo de campo en El Corcovo (Figura 1) permitió focalizar

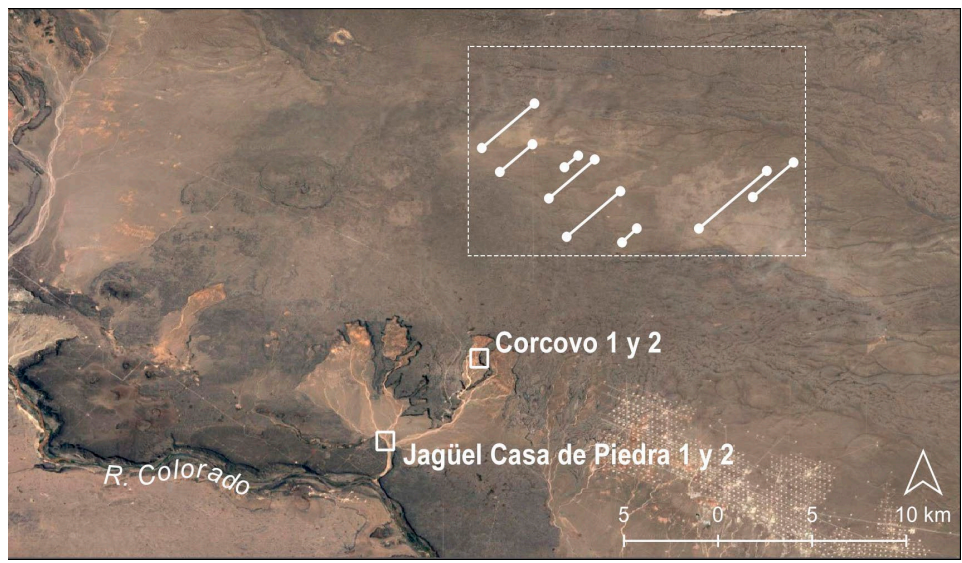
prospecciones en áreas actualmente ocupadas por pobladores. Los estudios permitieron informar sobre la existencia de sitios superficiales entre los que se mencionaron campamentos de actividades múltiples, en algunos casos, asociados con arte rupestre. Se registraron concentraciones de materiales arqueológicos en zonas de aguadas y vertientes. Entre esos sitios se destacó, por ser una evidencia sin precedente en el Sur de Mendoza, un conjunto de sitios superficiales asociados a petroglifos, los cuales se encuentran a escasos 1000 metros del actual Puesto Díaz (Localidad arqueológica Puesto Díaz- Figura 2). Pocos kilómetros, al Suroeste, se registró la localidad Jagüel Casa de Piedra emplazada casi inmediatamente al lado de un puesto abandonado. Ambas localidades conforman el sector arqueológico "El Corcovo" (Figura 2). Este trabajo informa los resultados obtenidos en trabajos de campo y laboratorio producidos desde 2005 hasta 2018. Sobre esa base se presentan las implicancias regionales de sus resultados principalmente en aspectos biogeográficos y dinámica poblacional.

Figura 2. Localización de los dos sectores y la zona transecteada (Tabla 1) en el área Corcovo: Localidad Puesto Díaz (sitios Corcovo-1 y Corcovo-2) y Localidad Jagüel Casa de Piedra (sitios Jagüel Casa de Piedra-1 y Jagüel Casa de Piedra-2).

Figure 2. Location of the two sectors and the surveyed area (Table 1) in the EI Corcovo area: Puesto Díaz locality (Corcovo-1 and Corcovo-2 sites) and Jagüel Casa de Piedra locality (Jagüel Casa de Piedra-1 and Jagüel Casa de Piedra-2 sites).

\section{Consideraciones Metodológicas y Técnicas}

Los trabajos de campo consistieron en transectas continuas para el relevamiento distribucional, excavaciones estratigráficas y relevamiento de arte rupestre. El relevamiento superficial consistió en transectas compuesta por unidades de ocho metros de ancho y 200 metros de longitud (Figura 2). La metodología del relevamiento fue pedestre y superficial, siguiendo los criterios aplicados en la región (Gil y Neme 2006). Durante dichas prospecciones se referenció, mediante el uso de GPS, la posición de materiales arqueológicos.

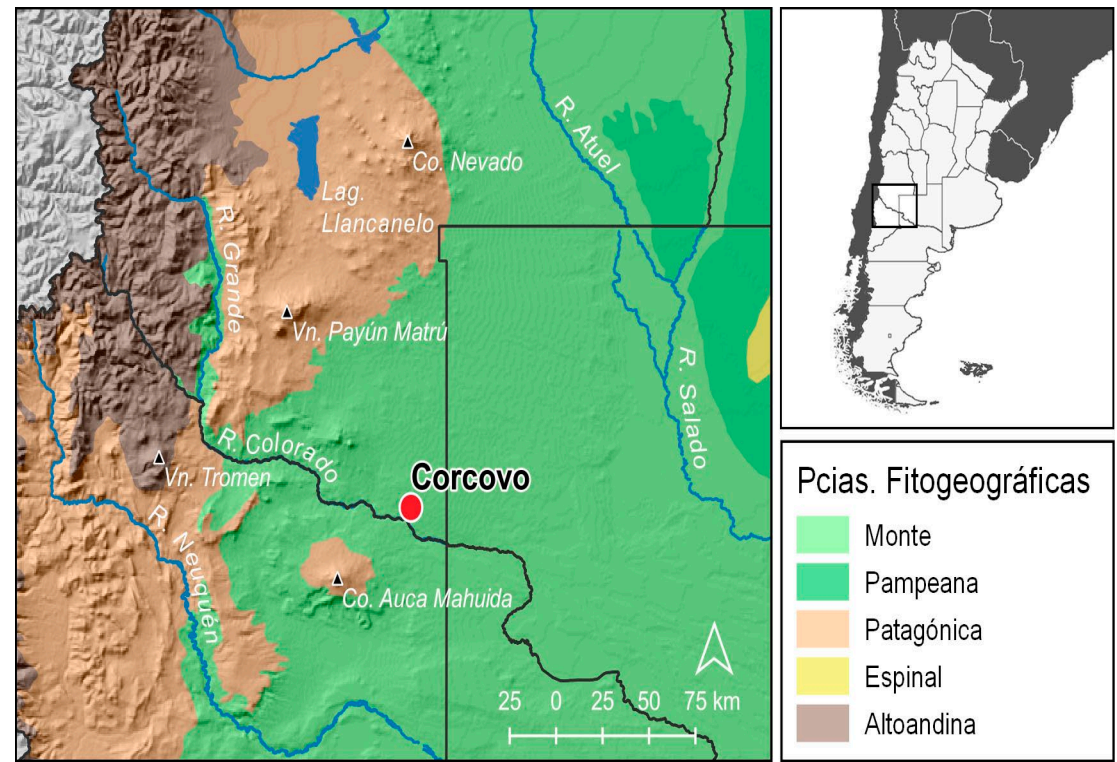

Figura 1. Localización del área El Corcovo en la macro-región.

Figure 1. Location of the El Corcovo area in the macro-region.
También se revisaron perfiles estratigráficos con el objetivo de ver las tasas de entierro de los materiales así como los procesos de formación intervinientes en cada lugar. Las principales variables del paisaje relevadas durante estas transectas fueron la pendiente y la visibilidad (Gil y Neme 2006).

En una de las concentraciones arqueológicas superficiales, denominada Corcovo-1 (Figura 2), se definió un área para excavar (unidad A1, $2 \mathrm{~m} \times 1 \mathrm{~m}$.). Los niveles de extracción fueron definidos por un espesor de $10 \mathrm{~cm}$. Todo el sedimento extraído se pasó por cernidor con malla de $2 \mathrm{~mm} 2$ obteniéndose, además, muestras en planta y muestras de sedimento para procesar por flotación en laboratorio. Los materiales 
separados en cernidor se embolsaron segregándose por sector y nivel. Se fotografiaron las plantas de cada nivel una vez que el mismo estaba terminado y se confeccionó a la vez una planilla con datos contextuales del mismo. La cronología estimada se basa en dos análisis radiocarbónicos realizados en Arizona AMS Facility sobre muestras de carbón disperso en capa.

El análisis tecnológico lítico se realizó siguiendo la propuesta de Aschero $(1975,1983)$ y Aschero y Hocsman (2004). Se consideraron en particular las variables: materia prima, medidas de tamaño máximo (largo, ancho y espesor), clases artefactuales (artefactos formatizados: unifaciales y bifaciales; desechos de talla y núcleos), tipo de artefactos, presencia-ausencia de corteza e índice de reducción (Ingbar et al. 1989). Para el tratamiento del conjunto lítico se tuvieron en cuenta los índices propuestos por Ericson (1984), que tienen como propósito generar información referente a los estadios de producción de una materia prima en particular. Se utilizaron: el índice de corteza (desechos primarios y secundarios de descortezamiento / $\mathrm{n}$ total de desechos) y el índice de producción (desechos de talla / instrumentos).

Para el análisis de los fragmentos cerámicos se continuó con la metodología aplicada en la región (Orton et al. 1993; Rye 1988; Sugrañes 2017a). En primera instancia, se procedió al remontaje de los fragmentos, a partir del cual se redujo el $\mathrm{N}$ de tiestos, dado que los fragmentos remontados se consideran como uno solo. Estos fragmentos fueron observados macroscópicamente y con lupa binocular (Microscopio Estereoscópico Nikon SMZ 800), a través de un corte en fresco para observar la pasta. Las variables relevadas y procesadas consisten en medidas métricas como ancho, largo, espesor y peso del fragmento y tamaño de inclusiones. Además, se tomaron el rasgo del fragmento, el tratamiento y color de la superficie, presencia de decoración, huellas de uso y post-depositacionales, características de la pasta (inclusiones y matriz) y tipo de cocción (Sugrañes 2017a).

Los restos arqueofaunísticos fueron identificados anatómica y taxonómicamente a partir del análisis comparativo con muestras de referencia actuales, principalmente de la colección del Museo de Historia Natural de San Rafael (MHNSR) y del Instituto Argentino de Zonas Áridas (IADIZA). Los mismos fueron posteriormente cuantificados empleando las medidas zooarqueológicas tradicionales y los criterios usados en la región (Neme y Gil 2002, 2008).

El registro de información del arte rupestre se realizó mediante planillas de relevamiento, fotografías y GPS. El llenado de las planillas se desarrolló siguiendo un protocolo específico de carga de datos (que define las variables a relevar y sus estados posibles), diseñado para alcanzar mayores niveles de consistencia y rigurosidad en el registro, reduciendo el margen de error asociado al operador. Una vez en el laboratorio, todas las fotografías fueron analizadas con el software DStretch-ImageJ (Harman 2008). Las variables que se presentarán en este trabajo, combinando observaciones en trabajo de campo y laboratorio, son las siguientes: a) a escala de sitio y de bloque: coordenadas, orientación cardinal, materia prima del soporte, dimensiones, tipo de topografía; b) a escala de motivo: grupo tipológico (conjunto de tipos de motivos que tienen afinidades morfológicas), técnica de ejecución, color, presencia de pátinas (clara, intermedia, oscura), estado de conservación (bueno, regular, malo).

La discusión de los resultados se enmarcan teniendo en cuenta un modelo demográfico generado sobre la base de fechados $14 \mathrm{C}$ de la región que en este caso incluye el Sur de Mendoza y Norte de Neuquén (Gil et al. 2014; Pérez et al. 2016). Impulsado desde el artículo pionero de Rick (1987), se explora la frecuencia de las fechas de radiocarbono arqueológicas a través del tiempo como un indicador de las altas y bajas en la dinámica de la población humana. Numerosos estudios han ido ajustando esta propuesta (Crema et al. 2017; Pérez et al. 2016; Shennan et al. 2013). Específicamente, utilizamos el paquete Rcarbon (Bevan y Crema 2018) para el análisis de grandes bases de datos de fechas radiocarbónicas, con especial énfasis en el enfoque de "fecha como datos" iniciado por Rick (1987). Así, se estiman la suma de densidad de probabilidades (SPD sus siglas en inglés, summed probability distributions). Para detalles ver Crema et al. (2017) y Bevan y Crema (2018).

\section{Resultados}

Se relevaron $188.800 \mathrm{~m} 2$ de transectas, según se detalla en Tabla 1 y Figura 2. En todos ellos se midieron las variables señaladas en metodología. Los resultados mostraron una baja depositación arqueológica superficial. Las prospecciones dirigidas, focalizadas en vertientes o lugares con disponibilidad de agua, se centraron en los siguientes Puestos: Puesto "Vidriera", Puesto "Moya" y Puesto "Luanco" y Puesto "Díaz". En todos ellos se encontraron alta densidad de materiales y amplias dispersiones.

En la localidad Puesto Díaz, los trabajos se centraron en la excavación del sitio a cielo abierto Corcovo-1 (Cor-1) y el relevamiento de los petroglifos adyacentes, denominados como sitio Corcovo-2 (Cor-2). En la localidad Jagüel Casa de Piedra, los trabajos se centraron en relevar el arte rupestre de dos aleros adyacentes denominados Jagüel Casa de Piedra-1 (JCP-1) y Jagüel Casa de Piedra-2 (JCP-2).

\section{Localidad Puesto Díaz}

Esta localidad incluye distintos sitios registrados originalmente en el año 2005. Por su relevancia en esta primera etapa de investigaciones en 2018 se dio 


\begin{tabular}{cccc}
\hline Transecta & Inicio & Fin & Superficie $\left(\mathrm{m}^{2}\right)$ \\
\hline 1 & $37^{\circ} 18^{\prime} 51.8^{\prime \prime}-68^{\circ} 23^{\prime} 5.9^{\prime \prime}$ & $37^{\circ} 17^{\prime} 17.6^{\prime \prime}-68^{\circ} 21^{\prime} 9.6^{\prime \prime}$ & 34400 \\
2 & $37^{\circ} 19^{\prime} 3.1^{\prime \prime}-68^{\circ} 21^{\prime} 5.4^{\prime \prime}$ & $37^{\circ} 18^{\prime} 45.6^{\prime \prime}-68^{\circ} 23^{\prime} 43.8^{\prime \prime}$ & 6400 \\
3 & $37^{\circ} 17^{\prime} 32.4^{\prime \prime}-68^{\circ} 23^{\prime} 44.4^{\prime \prime}$ & $37^{\circ} 16^{\prime} 11.9^{\prime \prime}-68^{\circ} 22^{\prime} 5.2^{\prime \prime}$ & 28800 \\
4 & $37^{\circ} 16^{\prime} 3.9^{\prime \prime}-68^{\circ} 22^{\prime} 40.8^{\prime \prime}$ & $37^{\circ} 16^{\prime} 27.9^{\prime \prime}-68^{\circ} 23^{\prime} 10.7^{\prime \prime}$ & 8000 \\
5 & $37^{\circ} 14^{\prime} 16.1^{\prime \prime}-68^{\circ} 24^{\prime} 15.6^{\prime \prime}$ & $37^{\circ} 15^{\prime} 48.2^{\prime \prime}-68^{\circ} 26^{\prime} 9.4^{\prime \prime}$ & 32000 \\
6 & $37^{\circ} 15^{\prime} 40.4^{\prime \prime}-68^{\circ} 24^{\prime} 19.8^{\prime \prime}$ & $37^{\circ} 16^{\prime} 37.6^{\prime \prime}-68^{\circ} 25^{\prime} 30.4^{\prime \prime}$ & 20000 \\
7 & $37^{\circ} 18^{\prime} 34.7^{\prime \prime} 68^{\circ} 18^{\prime} 20^{\prime \prime}$ & $37^{\circ} 16^{\prime} 35.4^{\prime \prime} 68^{\circ} 15^{\prime} 53.0^{\prime \prime}$ & 29600 \\
8 & $37^{\circ} 17^{\prime} 29.6^{\prime \prime} 68^{\circ} 16^{\prime} 23.5^{\prime \prime}$ & $37^{\circ} 16^{\prime} 18^{\prime \prime} 68^{\circ} 14^{\prime} 55.2^{\prime \prime}$ & 29600 \\
\hline
\end{tabular}

Tabla 1. Localización de las transectas y superficie relevada en cada una de ellas.

Table 1. Location of the transects and surface surveyed in each one of them. prioridad a los Cor-1 y Cor-2 (Figura 3A y B).

\section{Sitio Corcovo-1 (Cor-1)}

El sitio Cor-1, que cubre aproximadamente $200 \mathrm{~m} 2$ de superficie (Figuras 3A y B), registra una distribución principalmente de materiales líticos de una diversidad de rocas entre las que se incluyen basalto, riolitas, sílice y obsidiana. Se excavó una cuadrícula (A-1) de 2 x $1 \mathrm{~m}$, que incluyó dos sectores (Norte y Sur) de 1 x $1 \mathrm{~m}$ cada uno. La profundización de esta cuadrícula se realizó según 12 extracciones de $10 \mathrm{~cm}$ de profundidad. La excavación se continuó profundizando a pesar de no existir evidencias arqueológicas con el fin de confirmar el inicio der las ocupaciones por ello se sostiene que las mismas se dan desde la extracción 12.
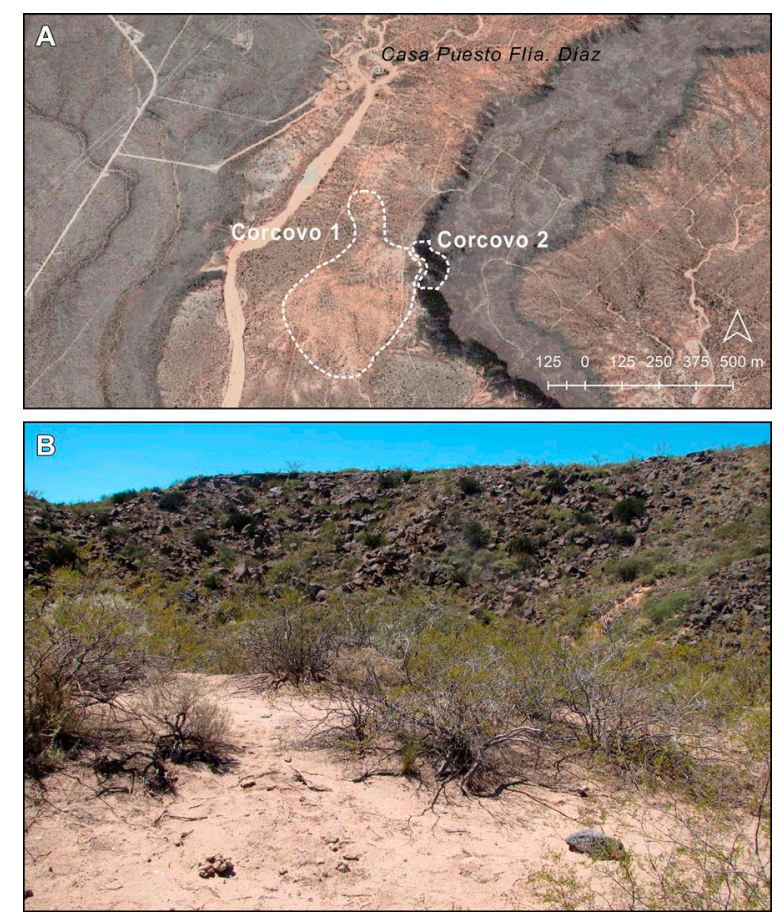

Figura 3. A) Vista del Sitio Cor-1 en relación al puesto actualmente ocupado por la Familia Díaz; B) Vista de materiales arqueológicos en sitio Cor-1 y detrás los bloques de basalto grabados de Cor-2.

Figure 3. A) View of the Cor-1 site in relation to the Puesto, actually occupied by Díaz Family; B) View of archaeological materials on site Cor-1 and, behind the engraved basalt blocks of Cor-2.
La matriz sedimentaria del depósito es homogénea, mayormente de arena. Hay una fuerte disminución en la cantidad de elementos líticos a medida que aumenta la profundidad. La misma tendencia se nota con los fragmentos de cáscaras de huevos de Rhea sp. Una tendencia distinta muestran los carbones que principalmente se registraron dispersos en capa. Los mismos notan una baja frecuencia inicial para incrementar hasta los niveles medios y declinar posteriormente hacia los niveles inferiores. Estas tendencias son semejantes a las observadas en otros depósitos excavados a cielo abierto en La Payunia (Gil 2006).

Se procesaron dos muestras de carbón disperso en capa para estimar la cronología radiocarbónica (Tabla 2). El análisis se realizó en Arizona AMS Facility (University of Arizona). La muestra superior proviene del Nivel 4, sector Sur, correspondiéndole $626 \pm 19$ años 14C AP ( $\delta 13 C$-24\%o) (AA-111425). Calibrada usando OXCal, curva SHCal-13 se obtiene una mediana de 605 años cal. AP (rango de 625 a 550 años cal AP, 68.2\%; 1345 años $A D$, rango 1325 a 1400 años $A D)$. La muestra inferior se extrajo en el nivel 12, sector Sur, arrojó como resultado $1878 \pm 23$ años 14C AP ( $\delta 13 C$-24\%o) (AA-111424). Calibrada del mismo modo que la fecha anterior, se estima en 1777 años cal AP (rango de 1816 a 1734 años cal. AP, 68.2\%; 174 años AD, rango 134 a 216 años AD). Estas dos fechas, junto a los cambios en la distribución estratigráfica de los materiales arqueológicos permitieron definir dos unidades temporales de análisis (Tabla 2): conjunto A (post 605 años cal. AP) y conjunto B (entre 605 y 1777 años cal AP).

El registro de arqueofauna incluye 9694 especímenes: fragmentos óseos, osteodermos y fragmentos de cáscaras de huevos (Tabla 3). Debido a la fuerte erosión y fragmentación, un alto número de restos no pudieron identificarse en una mayor resolución anatómica ni taxonómica. El NTAXA es de seis, todas registradas en conjunto $A$, y de ellas, cuatro en el conjunto B. Son abundantes en ambos conjuntos los fragmentos de cáscaras de huevos de Rhea sp. (Conjunto $A=80 \%$, Conjunto $B=64 \%$ del total de restos zooarqueológicos). El resto de los taxa identificados en Cor-1 son armadillos (C. vellerosus, C. villosus, Zaedyus pichiy), rodentia (Galea 
Tabla 3. Abundancia taxonómica por conjunto. Valores entre paréntesis indica número de osteodermos o fragmento de cáscadras de huevo; valores entre corchetes indica cantidad en gramos.

Table 3. Taxonomic abundance per set. Values in parentheses indicate number of osteoderms or the number of eggshell fragments and values in square brackets indicate quantity in grams.

\begin{tabular}{ccc}
\hline Variables & Conjunto $A$ & Conjunto $B$ \\
\hline Años ${ }^{14} \mathrm{C}$ AP & $626 \pm 19$ & $1878 \pm 22$ \\
Años cal AP & 605 & 1777 \\
Años cal AD & 1345 & 174 \\
Años de depósito* & {$[475](455)$} & {$[1250](1172)$} \\
Lítico $(\mathrm{n})$ & 500 & 212 \\
Tasa temporal lítico $\left((\mathrm{n} / \mathrm{años})^{*} 100\right)$ & 125 & 16,96 \\
Tasa depositacional lítico $\left[\left((\mathrm{n} / \mathrm{años})^{*} 100\right) / \mathrm{m}^{2}\right]$ & 62,5 & 8,48 \\
Óseo $(\mathrm{n})$ & 8014 & 1680 \\
Tasa temporal óseo $\left((\mathrm{n} / \mathrm{años})^{*} 100\right)$ & 2000 & 134 \\
Tasa depositacional óseo $\left[\left((\mathrm{n} / \mathrm{años})^{*} 100\right) / \mathrm{m}^{2}\right]$ & 1000 & 67 \\
Cerámico $(\mathrm{n})$ & 9 & 0 \\
Tasa temporal cerámico $\left((\mathrm{n} / \mathrm{años})^{*} 100\right)$ & 2.25 & 0 \\
Tasa depositacional cerámica $\left[\left((\mathrm{n} / \mathrm{annos})^{*} 100\right) / \mathrm{m}^{2}\right]$ & 1.12 & 0 \\
\hline
\end{tabular}

sp.), y guanacos (L. guanicoe). Estas taxas tienen un NISP muy bajo y significativamente menor al del recurso mayoritario previamente mencionado. Diferenciándose del conjunto $A$, el conjunto $B$ no registra $C$. vellerosus ni Galea sp. Considerando la tasa temporal, es mayor la depositación de cáscaras de huevos en el conjunto $A$, respecto al conjunto $B(18: 1)$. En los otros taxa donde esta comparación inter conjunto fue posible realizar (C. villosus, Z. pichiy y L. guanicoe) se registra la misma tendencia aunque con valores menores al anteriormente mencionado, entre 7:1 y 10:1.

Luego del remontaje, la cantidad de tiestos cerámicos sumó nueve, registrados entre el nivel 1 y 4 (Tabla 2; Figura 4 A, B y C), asignándose todos al conjunto $A$. El remontaje de tres fragmentos distribuidos en los tres primeros niveles de excavación sugiere que estos pueden reflejar un promedio de ocupación, señalando una baja resolución estratigráfica del depósito. El conjunto cerámico se compone de fragmentos con tamaños pequeños que no superan los $5 \mathrm{~cm}$ de lado. El espesor promedio de la muestra es de $5.9 \mathrm{~mm}$, siendo el mínimo $3.7 \mathrm{~mm}$ hasta un máximo de $7.9 \mathrm{~mm}$. Los fragmentos se encuentran en buen estado de conservación, siendo sólo afectados por quebramiento y laminado en sus superficies alisadas. Este último es el tratamiento predominante. Sólo dos fragmentos poseen pulido en ambas caras, siendo uno de ellos un borde fino y recto que puede asociarse a una vasija de boca no restringida. Se diferencian dos tipos de pastas: uno predominante con presencia de inclusiones finas a gruesas con un pobre ordenamiento y contorno subangular, con abundante presencia de cuarzo irregular; el otro corresponde a los fragmentos pulidos y consiste en inclusiones finas con un ordenamiento equilibrado y de contorno redondeado. Las tipologías (Sugrañes y Franchetti 2012; Sugrañes 2017a) que se determinaron corresponden principalmente a Overo/Nihuil (Figura 4C) en un $55,5 \%(n=5)$ del conjunto, luego a Atuel (Figura $4 A)$ en un $22,2 \%(n=2)$, mientras que un $22,2 \%(n=$ 2) se mantienen indeterminados correspondiendo a los fragmentos pulidos (Figura 4B) con atributos similares a cerámicas del norte de Neuquén. De acuerdo a lo anterior, puede establecerse que los fragmentos de la excavación representan un número mínimo de tres vasijas.

Las características de las vasijas muestran la posibilidad

Tabla 4. Materias primas y clases artefactuales según conjuntos A y B. AF bif: artefacto formatización bifacial; AF Unif: artefacto formatización unifacial; DT: desecho de talla; Nuc: núcleo.

Table 4. Raw material and artefactual classes according to sets $A$ and $B$.

\begin{tabular}{lll} 
& Conjunto A & Conjunto B \\
\hline Vert. Indeter. & 1301 & 473 \\
Microvertebrado & 10 & 1 \\
Ave media & 8 & 10 \\
Rheidae & $(6440)$ [478] & $1(1074)$ [95] \\
Mam. Indet & 70 & 28 \\
Mam. mediano & 4 & 4 \\
Mam. grande & 1 & 0 \\
Micromamifero & 10 & 17 \\
Cingulata & $5(54)$ & $2(34)$ \\
Euphractinae & $(5)$ & $(5)$ \\
C. vellerosus & $(2)$ & 0 \\
C. villosus & $(5)$ & $(2)$ \\
Zaedyus pichiy & $(94)$ & $(27)$ \\
Galea sp. & 1 & 0 \\
L. guanicoe & 4 & 2 \\
\hline Total & 8014 & 1680
\end{tabular}


Tabla 4. Materias primas y clases artefactuales según conjuntos A y B. AF bif: artefacto formatización bifacial; AF Unif: artefacto formatización unifacial; DT: desecho de talla; Nuc: núcleo.

Table 4. Raw material and artefactual classes according to sets $A$ and $B$.

\begin{tabular}{|c|c|c|c|c|c|c|c|c|c|c|c|c|c|c|c|c|c|c|c|c|}
\hline \multirow[b]{2}{*}{ 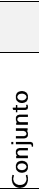 } & \multirow[b]{2}{*}{$\stackrel{\bar{d}}{z}$} & \multicolumn{4}{|c|}{ Rocas Silíceas } & \multicolumn{4}{|c|}{ Obsidiana } & \multicolumn{2}{|l|}{ Riolita } & \multicolumn{2}{|c|}{ Basalto } & \multicolumn{2}{|l|}{ Dacita } & \multicolumn{2}{|c|}{ Cuarzo } & \multirow{2}{*}{$\begin{array}{c}\text { Arenisca } \\
\\
5\end{array}$} & \multirow{2}{*}{$\begin{array}{r}\text { Toba } \\
\text { 占 }\end{array}$} & \multirow[b]{2}{*}{$\begin{array}{l}\bar{\pi} \\
0 \\
0\end{array}$} \\
\hline & & $\begin{array}{l}\frac{\omega}{0} \\
\frac{u}{<}\end{array}$ & $\begin{array}{l}\text { 岁 } \\
\text { 岁 } \\
\text { 岁 }\end{array}$ & 5 & ’ & $\begin{array}{l}\frac{\omega}{\infty} \\
\frac{1}{<}\end{array}$ & $\begin{array}{l}\frac{u}{2} \\
\frac{1}{2} \\
\frac{u}{<}\end{array}$ & เ & ๖ & 5 & ’ & 5 & そ & 5 & $\stackrel{\text { ๖ }}{z}$ & そ & 5 & & & \\
\hline \multirow{3}{*}{$\varangle$} & $n$ & 7 & 3 & 249 & 2 & 4 & 2 & 121 & 1 & 84 & 1 & 17 & - & 6 & - & - & 2 & 1 & - & 500 \\
\hline & $\%$ & 1.4 & 0.6 & 49.8 & 0.4 & 0.8 & 0.4 & 24.2 & 0.2 & 16.8 & 0.2 & 3.4 & 0 & 1.2 & 0 & 0 & 0.4 & 0.2 & 0 & \\
\hline & $\%$ & \multicolumn{4}{|c|}{52} & \multicolumn{4}{|c|}{26.6} & \multicolumn{2}{|c|}{17} & \multicolumn{2}{|c|}{3.4} & \multicolumn{2}{|c|}{1.2} & \multicolumn{2}{|c|}{0.4} & 1 & 0 & 70 \\
\hline \multirow{3}{*}{$\infty$} & $\mathrm{n}$ & 1 & 3 & 87 & 1 & - & - & 56 & - & 46 & - & 11 & 1 & 1 & 1 & 1 & 1 & 1 & 1 & 212 \\
\hline & $\%$ & 0.5 & 1.4 & 41.4 & 0.5 & 0 & 0 & 26.4 & 0 & 21.7 & 0 & 5.2 & 0.5 & 0.5 & 0.5 & 0.4 & 0.5 & 0.5 & 0.5 & \\
\hline & $\%$ & \multicolumn{4}{|c|}{43} & \multicolumn{4}{|c|}{26.4} & \multicolumn{2}{|c|}{2} & \multicolumn{2}{|c|}{5.7} & \multicolumn{2}{|c|}{1} & \multicolumn{2}{|c|}{0.9} & 0.5 & 0.5 & 30 \\
\hline
\end{tabular}

de una variedad de funciones en la cerámica, a pesar de tan bajo registro. Para las vasijas alisadas, si bien con un solo fragmento con evidencias de uso, puede establecerse la cocción como principal función. En el caso de los fragmentos pulidos, pueden vincularse al almacenamiento, principalmente de agua, dada su impermeabilización de las superficies. Es decir, el registro cerámico de Cor-1 indicaría que las vasijas habrían estado, al menos funcionalmente, asociadas a la cocción y almacenamiento. Por otro lado, tanto los espesores, como el borde y base presentes, señalarían vasijas pequeñas, las cuales en esta región están asociadas a las ocupaciones de cazadores-recolectores (Sugrañes 2017a y b).

El análisis lítico incluyó la totalidad del material recuperado, 712 artefactos. Predominan los artefactos trabajados sobre roca silícea (50\%), seguido por obsidiana (23\%), riolita (21\%), basalto (4\%), dacita $(2 \%)$, cuarzo $(0.6 \%)$ y otras $(0.7 \%)$. En la tabla 4 se destaca que la mayor variedad de tipos artefactuales se presenta en rocas silíceas y obsidiana, mientras que en las restantes materias primas sólo se identificaron desechos de talla y núcleos. El conjunto A incluye 500 artefactos y en el conjunto $B$ se registran 212 . Si bien la tasa temporal de depositación de materiales líticos es significativamente mayor en el conjunto $A$ en relación al B (Tabla 3), en este último se registra mayor diversidad de materias primas (Tabla 5).

En el conjunto A los instrumentos $(\mathrm{N}=16)$ están trabajados tanto sobre rocas silíceas (63\%) como obsidiana (37\%) y el $81 \%$ se encuentra fracturado. Mientras, en el conjunto $B(\mathrm{~N}=4)$ están confeccionados exclusivamente en rocas silíceas y el $50 \%$ se encuentra fracturado. En el conjunto $A$, el $69 \%(N=11)$ corresponde a artefactos de formatización bifacial, uno entero (punta de proyectil triangular con pedúnculo convexo convergente confeccionada en roca silícea) y diez fracturados. Entre los fracturados, hay seis en rocas silíceas (cuatro ápices de punta de proyectil y dos artefactos no diferenciados) y tres en obsidiana (tres bases de punta de proyectil y un artefacto no diferenciado por fractura). El modulo tamaño de los instrumentos enteros en A es medianopequeño (67\%) y pequeño (33\%). En el conjunto B el $25 \%$ está representado por un artefacto de formatización bifacial (ápice de punta de proyectil con morfología no diferenciada por fractura), mientras que el $75 \%$ de los instrumentos pertenecen a artefactos unifaciales (dos raspadores y un artefacto de formatización unifacial no diferenciado por fractura). El tamaño de los instrumentos enteros en $\mathrm{B}(\mathrm{N}=2)$ es muy grande y grandísimo.

En el conjunto $A$ los núcleos $(\mathrm{N}=4)$ son dos de rocas silíceas, uno de riolita y uno de obsidiana; el núcleo de obsidiana y uno de sílice no poseen morfología definida, su tamaño es mediano-pequeño y se encuentran agotados. El núcleo de riolita presenta lascados aislados, tamaño muy grande y no está agotado, mientras que el restante de sílice se encuentra fracturado. En el conjunto B los núcleos $(\mathrm{N}=4)$ son de basalto, dacita, cuarcita y rocas silíceas; los dos primeros presentan lascados aislados, tamaño muy grande y no están agotados. Por su parte, el núcleo de cuarcita se encuentra fracturado, mientras que el núcleo trabajado en sílice presenta una morfología subpiramidal, tamaño pequeño y se encuentra agotado.

Respecto a las etapas de reducción, en el conjunto A se identificó la presencia de todas las etapas de reducción sobre rocas silíceas, basalto, obsidiana y riolita (Figura 4D). También se registran las etapas iniciales y finales de la secuencia de la dacita. En arenisca y cuarcita sólo hay lascas internas. Se destaca una mayor frecuencia en las etapas finales en rocas silíceas y basalto. En la Figura 4D se observa que en el conjunto B se encuentran representadas también todas las etapas de la secuencia de reducción en rocas silíceas, basalto, obsidiana, riolita, y sólo lascas internas en dacita, toba y cuarcita. Se resalta la presencia de lascas externas de obsidiana y de 


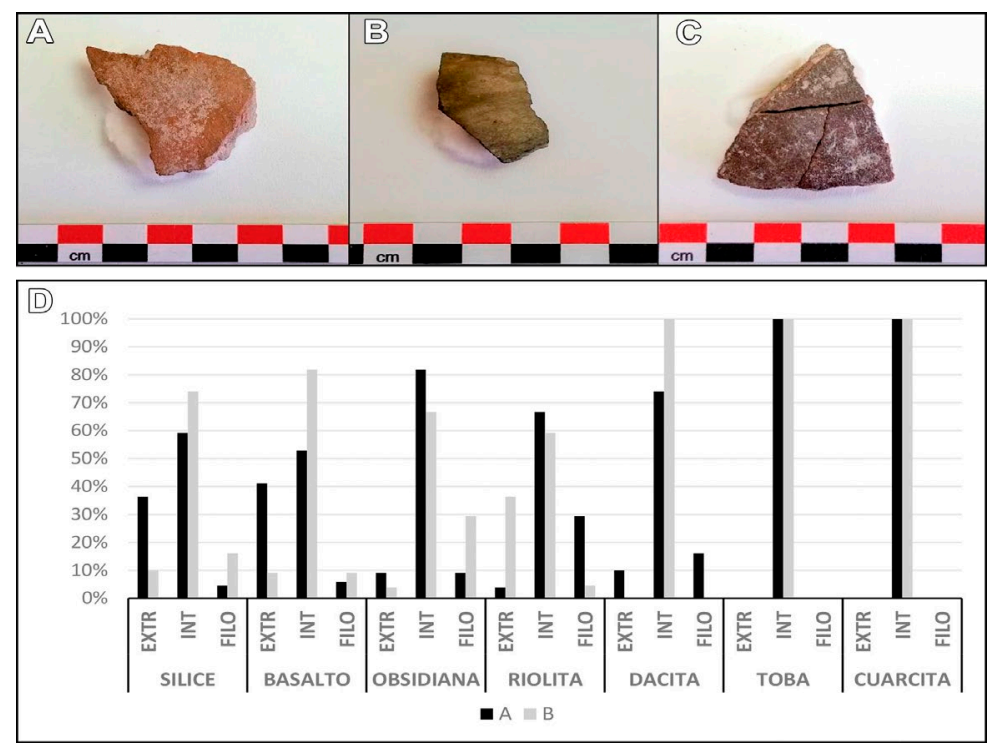

Figura 4. A) Tiesto cerámico Atuel; B) Tiesto cerámico Indeterminado pulido; C) Tiesto cerámico Nihuil/Overo; D) Secuencia por materia prima lítica comparativa entre conjunto; ext: externa; int: interna; $A$ : conjunto $A, B$ : conjunto $B$.

Figure 4. A) Atuel ceramic sherd; B) Polish unknown ceramic sherd; C) Nihuil/Overo ceramic sherd; D) Sequence by comparative raw material between sets. la intensidad de uso de las materias primas y la secuencia de manufactura de los artefactos. En la tabla 6 se observa que las rocas silíceas y obsidiana son las materias primas que presentan mayor valor medio de densidad de extracciones en ambos subconjuntos, siendo mayores para el conjunto A. Asimismo, en B, se destaca el valor de índice de extracciones sobre basalto que es mayor al observado en conjunto A (Tabla 6). En riolita no se observan diferencias en los valores de índice y hay escasa variabilidad en la densidad de extracciones. En relación a los valores mínimos y máximos en el número de extracciones se observa que todas las materias primas han ingresado al sitio con corteza y que los valores máximos aumentan en casi todas las materias primas del conjunto $B$ al $A$, a excepción del basalto que disminuye. Se destacan los valores máximos de la obsidiana que se duplica en $A$, indicando una mayor intensidad de uso de esta materia prima en éste conjunto. formatización de filo en riolita.

En la tabla 5 se presentan los valores para el índice de corteza propuesto por Ericson (1984). Este índice es indicativo de cómo ingresa la materia prima al sistema de producción lítica. En el conjunto A, las materias primas ingresan con valores de índice mayor en todos los casos, por lo que, se puede plantear que en B el ingreso de la materia prima al sistema se daba en forma de nódulos sin procesar, destacando la diferencia en los valores de basalto y obsidiana. Del mismo modo, en la relación entre desechos de talla e instrumentos notamos valores superiores en $A$ en relación a $B$, sobre todo en obsidiana.

Para estimar el grado de reducción, se calcula el valor medio de la cantidad de extracciones previas en la cara dorsal de los desechos de talla enteros (exceptuando las lascas de formatización de filo) por milímetro cuadrado (extracciones por mm2 o e/mm2) (Ingbar et al. 1989; Ingbar 1994). Este es un indicador útil para estimar Tabla 5. Índice de corteza y de producción (sensu Ericson 1984).

Table 5. Cortex and production index (sensu Ericson 1984).

\begin{tabular}{lllll}
\hline COR-1 & \multicolumn{2}{l}{ Índice de Corteza } & \multicolumn{2}{l}{ Índice de Producción } \\
\hline Materia Prima & Conjunto A & Conjunto B & Conjunto A & Conjunto B \\
\hline Rocas Silíceas & 0.18 & 0.09 & 24.90 & 21.75 \\
Basalto & 0.41 & 0.09 & 0.00 & 0.00 \\
Obsidiana & 0.21 & 0.04 & 20.17 & 0.00 \\
Dacita & 0.14 & 0.00 & 0.00 & 0.00 \\
Riolita & 0.24 & 0.15 & 0.00 & 0.00 \\
\hline
\end{tabular}

En el registro lítico se destaca el uso de materias primas locales, como son las rocas silíceas (Salgán 2013, 2015) y los rodados de rocas disponibles en el río Colorado, como son el basalto, la obsidiana y la riolita. La presencia de toda la secuencia de reducción en obsidiana apoya la propuesta de la disponibilidad secundaria de esta roca en la cuenca de los ríos Barrancas y Colorado (Salgán 2013), recientemente confirmada en la cuenca alta del río Barrancas por Fernández et al. (2017). Sin embargo, el uso de estos recursos fue diferente en los dos conjuntos. En el conjunto $A$, los instrumentos están trabajados en rocas silíceas y obsidiana y, en ambas materias primas, los artefactos bifaciales son más frecuentes que los unifaciales. En desechos de talla se registran todas las etapas de reducción en sílice, obsidiana, basalto, riolita y dacita, al mismo tiempo que se registra un incremento en la intensidad de uso de las materias primas más frecuentes. En el conjunto B, los artefactos formatizados documentados están trabajados exclusivamente en rocas silíceas, sin embargo, se identificaron todas las etapas de la secuencia de reducción en los desechos de talla de sílice, obsidiana, basalto y riolita. En términos de Ericson (1984), se observan diferencias en el sistema de producción lítica entre ambos conjuntos. En el conjunto A, el sistema de producción lítica puede atribuirse a un tipo terminal, donde los procesos de producción lítica se dan en un área específica, dentro del rango de distancia local. Por el contrario, en B el sistema puede atribuirse a un tipo secuencial, donde las diferentes etapas de reducción se dan en diferentes áreas.

Las diferencias observadas entre los conjuntos A y B 
Tabla 6. Estadística descriptiva de la densidad de extracciones previas en desechos de talla según conjunto y materia prima.

Table 6. Descriptive statistics for density of waste flake in debitage according to sets and raw material.

\begin{tabular}{lllllllll}
\hline COR-1 & Materia Prima & $\mathbf{N}$ & Media & Mediana & $\begin{array}{l}\text { Desvío } \\
\text { Estándar }\end{array}$ & Mínimo & Máximo & Rango \\
\hline \multirow{4}{*}{ A } & Silíceas & 50 & 1.99 & 1.49 & 1.86 & 0 & 8.38 & 8.38 \\
& Basalto & 13 & 0.66 & 0.28 & 1.03 & 0 & 3.56 & 3.56 \\
& Obsidiana & 85 & 1.72 & 1.53 & 1.44 & 0 & 9.38 & 9.38 \\
& Riolita & 71 & 0.80 & 0.55 & 0.86 & 0 & 4.09 & 4.09 \\
& Siliceas & 54 & 1.89 & 1.67 & 1.23 & 0 & 5.68 & 5.68 \\
& Basalto & 8 & 1.72 & 1.47 & 1.28 & 0 & 3.92 & 3.92 \\
& Obsidiana & 25 & 1.69 & 1.40 & 1.16 & 0 & 4.74 & 4.74 \\
& Riolita & 39 & 0.86 & 0.59 & 0.84 & 0 & 3.50 & 3.51 \\
\hline
\end{tabular}

pueden responder a cambios en la forma de ocupación del espacio que se dieron en los últimos 600 años respecto al uso inicial de esta localidad, desde ca. 1900 años AP. Para las ocupaciones que generaron el registro del conjunto $B$ se plantea una mayor movilidad residencial, ocupación menos frecuente y/o por menor tiempo. Por el contrario, el conjunto A habría sido producto de ocupación/nes de tipo residencial (mayor cantidad de tiempo y personas). Esta alternativa da cuenta de las diferencias observadas en los conjuntos líticos, principalmente en las observadas sobre aquellas rocas de mejor calidad para la talla, como son las silíceas y la obsidiana. Asimismo, la tasa de depositación temporal de materiales líticos en el conjunto $A$ es casi ocho veces mayor que en el conjunto $B$ (Tabla 3 ).

Sitio Corcovo-2 (Cor-2)

El sitio Cor-2 consiste en un conjunto de bloques basálticos contiguos con arte rupestre. Se emplaza en forma inmediatamente adyacente a Cor-1 e incluso podrían considerarse parte del mismo sitio. El arte rupestre de Cor-2 se encuentra emplazado sobre bloques rocosos de diferentes tamaños distribuidos dentro de una sección acotada de talud de borde de meseta orientado en sentido general Norte-Sur (Figura 3 y 5A). En el relevamiento se registró una superficie aproximada de $31.200 \mathrm{~m} 2(120 \times 260 \mathrm{~m})$, comprendida entre cotas de 720 y 760 msnm (Figura 3 y 5A). La delimitación de esta sección de talud para su relevamiento sistemático se basó en la información proveniente tanto de pobladores locales como de trabajos arqueológicos previos, que señalaban este espacio como el área de dispersión de los bloques con arte rupestre.

Se identificaron 71 bloques con arte rupestre (Figura 5A). Sobre las superficies de estos bloques se registró la presencia de un total de 83 paneles o "caras" con motivos rupestres. Esto implica que en la mayoría de los casos ( $\mathrm{N}=61 ; 86 \%)$ se utilizó solo una de las múltiples "caras" disponibles por bloque para la ejecución de imágenes. Sólo en 10 (14\%) de los 71 bloques relevados se identificó la presencia de motivos rupestres en más de una de sus "caras" (dos caras: $\mathrm{N}=8$ bloques; tres caras: $\mathrm{N}=2$ bloques).

En los 71 bloques que componen el sitio, se identificaron un total de 151 motivos. Una característica general del arte rupestre de Cor-2 consiste en la producción de pocos motivos por bloque. La muestra está dominada por bloques con un único motivo ( $\mathrm{N}=34 ; 47.8 \%)$, mientras que $19(26,8 \%)$ de los bloques registrados presenta dos motivos (Tabla 7).

Una caracterización morfológica general del arte rupestre de Cor-2 permite definirlo como una producción en la que predominan las figuras geométricas curvilíneas, aunque con una importante diversidad formal. El total de motivos registrados es de 151 y el repertorio de grupos tipológicos consta de 14 grupos de motivos, que incluyen: figuras geométricas curvilíneas simples y complejas, figuras geométricas "mascariformes" (sensu Gradin 1997/1998: 14), líneas rectilíneas simples y complejas, pisadas de animales, líneas curvilíneas simples, figuras geométricas complejas rectilíneas, etc. (Figura 5B-G).

En términos técnicos, se observa que la diversidad morfológica señalada no se traslada a las técnicas de ejecución de las imágenes rupestres. El arte de Cor-2 está producido exclusivamente mediante dos variedades técnicas de grabado: raspado $(\mathrm{N}=96 ; 63.6 \%)$, piqueteado $(\mathrm{N}=48 ; 32 \%)$ y la combinación entre ambas $(\mathrm{N}=7 ; 5 \%)$.

Resulta necesario destacar que el estado de conservación de los motivos registrados oscila frecuentemente entre regular y malo $(\mathrm{N}=93 ; 61 \%)$, siendo afectados 
principalmente por agentes de deterioro de origen abiótico (pátinas: $\mathrm{N}=65 ; 43 \%$ ), biótico (líquenes: $\mathrm{N}=12$; $8 \%$ ) y/o la combinación de ambos (líquenes y pátinas: $\mathrm{N}=36 ; 24 \%$ ), cuyo resultado repercute profundamente

Tabla 7. Frecuencia de motivos rupestres por bloques.

Table 7. Frequency of rock motifs by blocks

\begin{tabular}{ccc}
\hline Frecuencia de motivos & N bloques & \% bloques \\
\hline 1 & 34 & 47.8 \\
2 & 19 & 26.8 \\
3 & 8 & 11.3 \\
4 & 4 & 5.6 \\
5 & 3 & 4.2 \\
6 & 1 & 1.4 \\
8 & 1 & 1.4 \\
10 & 1 & 1.4 \\
Total & 71 & 100 \\
\hline
\end{tabular}
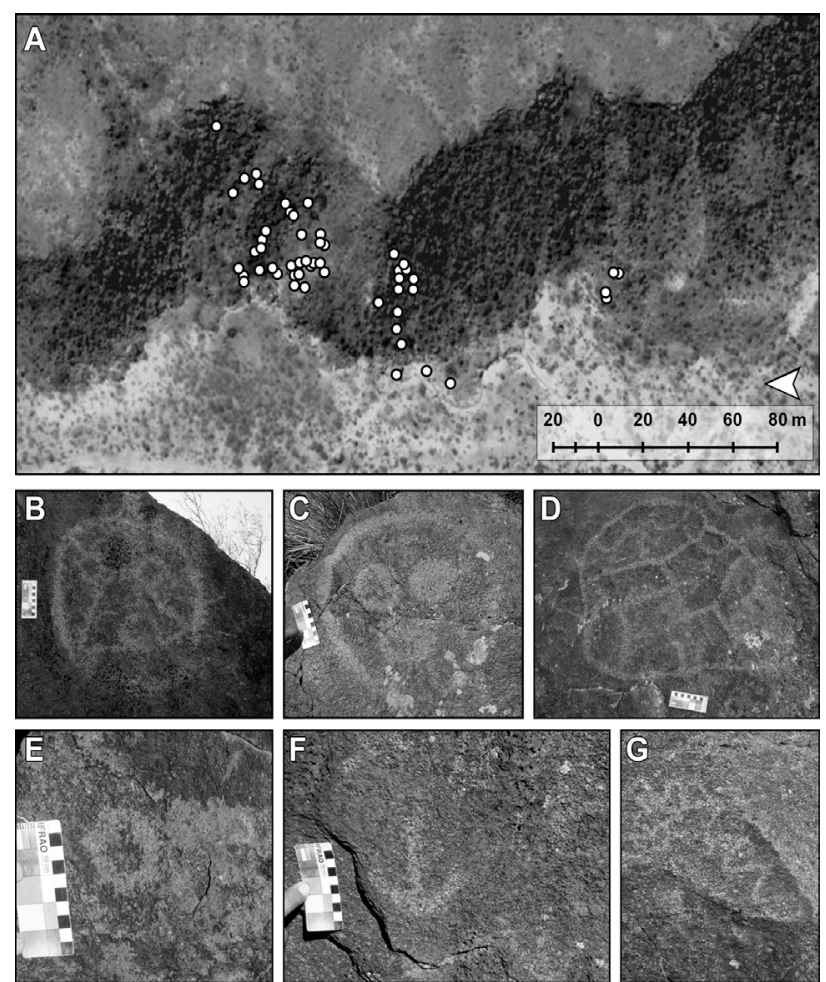

Figura 5. A) Distribución de los bloques con arte del sitio Corcovo-2; B y C) Figuras geométricas "mascariformes"; D) Figura geométrica curvilínea compleja; E) Figura geométrica curvilínea simple; F) Tridígitos; G) Línea rectilínea simple.

Figure 5. A) Distribution of blocks with rock art from the Corcovo-2 site; $B$ and C) "Mascariform" geometric figures; D) Complex curvilinear geometric figures; E) Simple curvilinear geometric figures; F) Threedigits (bird footprint); G) Simple rectilinear line. en la baja visibilidad de los motivos relevados.

\section{Localidad Jagüel Casa de Piedra.}

Esta localidad, emplazada a unos $7 \mathrm{kmts}$. de Puesto Díaz, está constituida por dos aleros: Jagüel Casa de Piedra-1 y Jagüel Casa de Piedra-2.

Se relevaron dos sitios con arte rupestre, muy cercanos entre sí, localizados sobre una barda de basalto orientada en sentido general Noroeste-Sureste: Jagüel Casa de Piedra-1 (JCP-1) y Jagüel Casa de Piedra-2 (JCP2) (Figuras 2 y 6A). Ambos sitios se encuentran ubicados a una altitud aproximada de $570 \mathrm{msnm}$. El sitio JCP-1 (S: $37^{\circ} 25^{\prime} 54.77^{\prime \prime}$ O: $68^{\circ} 29^{\prime} 37.98^{\prime \prime}$ ) consiste en una pequeña cueva de $2,4 \mathrm{~m}$ de ancho por 1,3 $\mathrm{m}$ de alto $\mathrm{y}$ $2,5 \mathrm{~m}$ de profundidad, orientada hacia el Noreste. Por su parte, JCP-2 (S: $37^{\circ} 25^{\prime}$ 54.60" O: 68 29'38.00") consiste en un pequeño alero de 2,5 $\mathrm{m}$ de ancho por 1,7 $\mathrm{m}$ de alto y 1,6 $\mathrm{m}$ de profundidad, también orientado hacia Noreste. Un rasgo importante de la producción rupestre de los dos sitios consiste en la utilización de, prácticamente, todas las superficies internas de la cueva o alero (paredes, techo y piso) para emplazar motivos rupestres.

El estado de conservación del arte rupestre en ambos sitios es malo, ya que está muy obliterado (término utilizado para remitir al deterioro de la morfología y/o de la intensidad del color de los motivos rupestres como consecuencia de factores naturales y/o antrópicos que afectan negativamente su preservación). Esto provoca que su visibilidad sea muy baja. Ello requirió del procesamiento, en el laboratorio, del total de las fotografías tomadas con el software DStretch-ImageJ. Utilizando esta información, los datos que aportamos en este trabajo son conteos mínimos de motivos, ya que es posible que otros motivos se hayan borrado por completo o que su identificación sea imposible incluso usando este software.

Cada motivo fue clasificado dentro de un grupo tipológico de acuerdo a la morfología de su diseño. El repertorio de JCP-1 consta, en su gran mayoría, de los siguientes grupos tipológicos de motivos: pisadas de animales (tridígitos y pisadas de felinos), líneas simples rectilíneas, figuras geométricas complejas rectilíneas, figuras geométricas simples rectilíneas y curvilíneas y figuras antropomorfas (Figura 6B). La mayoría de los motivos registrados en JCP-1 fueron producidos mediante pintura positiva (número mínimo de motivos= 81), grabado $(\mathrm{NMM}=2)$ y grabado repintado (NMM= 31). En cuanto al uso del color, el tono predominante es el rojo (NMM=69), aunque también se registran pinturas en amarillo pardo (NMM=7), blanco (NMM= 2) y combinaciones de dos colores (bicromas) rojas y amarillas (NMM=34). Resulta interesante destacar que, mientras la técnica de pintura se utilizó mayoritariamente para la realización de motivos geométricos, la técnica de 
grabado se utilizó casi exclusivamente para producción de pisadas de animales.

Cabe notar que en asociación con motivos grabados de pisadas aparecen pequeñas oquedades (tipo mortero o tacitas de tamaños variados) con su interior pulimentado, ubicados en el piso de la cueva y orientados hacia arriba (Figura 6D y E). Estas oquedades se agrupan en dos conjuntos. Uno de ellos incluye seis oquedades de forma circular de un diámetro promedio de $10 \mathrm{~cm}$, algunos de los cuales presentan restos de pigmento rojo en su interior, indicando la posibilidad de que hayan sido utilizados para moler pigmentos, sin descartar que hayan estado decorados. En el segundo conjunto, se encuentran cuatro morteros alineados, con un diámetro promedio de $10 \mathrm{~cm}$; en este caso no poseen evidencias de pigmento. Es esperable que estos morteros sean parte del contexto rupestre, tanto en su potencial uso funcional para moler pigmentos y/o como de potenciales actividades decorativas/performativas (véase Schobinger (2002) respecto de esta asociación entre arte y morteros en Mendoza).

Los datos registrados demuestran que el grado de
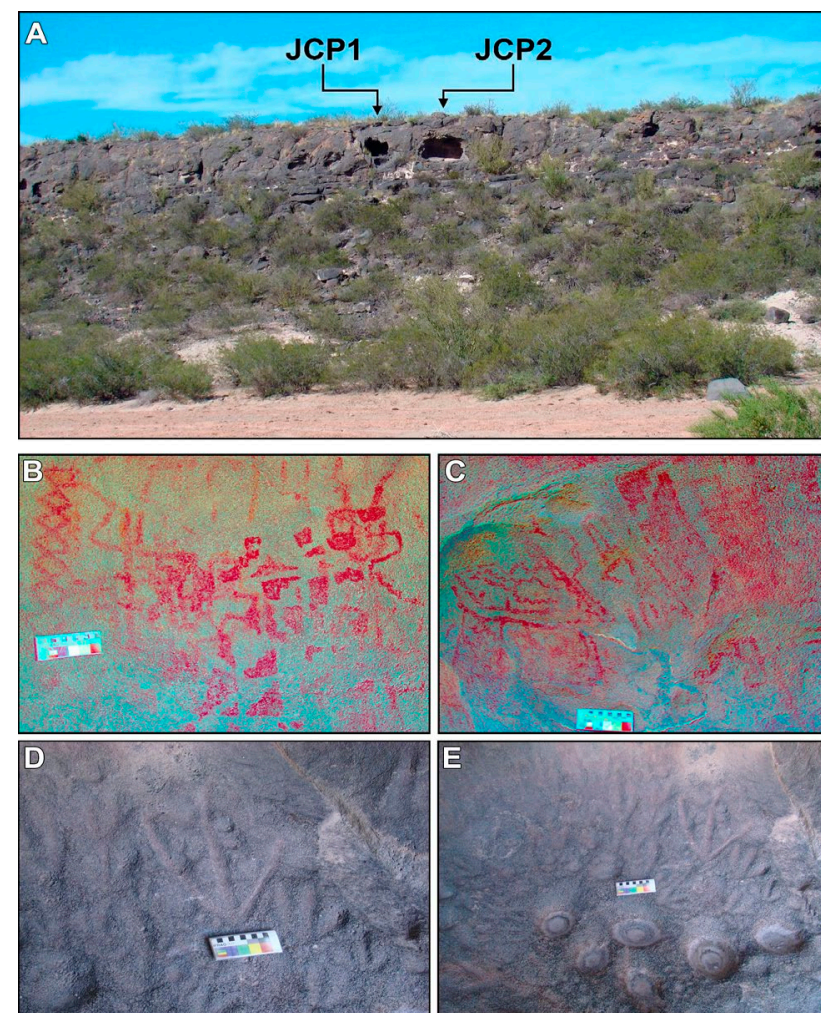

Figura 6. A) Sitios JCP-1 y JCP-2; B) Figuras geométricas rectilíneas complejas de JCP-1; C) Figuras geométricas rectilíneas complejas de JCP-2; D) Tridígitos grabados repintados de JCP-1; E) Oquedades asociadas a motivos grabados en JCP-1.

Figure 6. A) Sites $J(P-1$ y $J(P-2 ; B)$ Complex rectilinear geometric figures of JCP-1; C) Complex rectilinear geometric figures of JCP-2; D) Engraving three-digits repainted of JCP-1; E) Cavities associated to engraved motifs in JCP-1. conservación de las pinturas es predominantemente malo, con un alto grado de deterioro y bajo grado de integridad de los motivos y de intensidad de color/ contraste de la pintura sobre la roca soporte. Los principales factores de deterioro registrados son la obliteración, la formación de pátinas y, con menor frecuencia, el descascaramiento o exfoliación del soporte.

El sitio JCP-2 tiene un número mínimo de 53 motivos, clasificados en 10 grupos tipológicos (Figura 6C). Estos grupos son de características similares a los de JCP-1, incluyendo: líneas simples rectilíneas, figuras geométricas complejas rectilíneas, líneas simples curvilíneas, líneas complejas rectilíneas, figuras geométricas complejas curvilíneas, etc. No se registra la presencia de motivos de pisadas. Todos los motivos identificados en JCP-2 fueron producidos mediante técnica de pintura, no registrándose ningún motivo grabado. Para la ejecución de los motivos pintados se seleccionó predominantemente el color el rojo (NMM= 43), en menor medida, combinaciones bicromas de rojo y amarillo $(\mathrm{NMM}=9)$ y amarillo $(\mathrm{NMM}=1)$.

Finalmente, el grado de conservación de las pinturas de JCP-2 es predominantemente malo, con un alto grado de deterioro y bajo grado de integridad de los motivos y de intensidad de color/contraste de la pintura sobre la roca soporte. Los principales factores de deterioro registrados son la obliteración y, en menor frecuencia, el descascaramiento o exfoliación del soporte.

\section{Discusión}

Los resultados obtenidos en estas primeras investigaciones muestran el inicio de la ocupación humana en El Corcovo en aproximadamente 1900 años 14C AP (cal. 1800 años AP). Asimismo, se obtuvo la cronología para el nivel a partir del cual se registró un cambio en la frecuencia de hallazgos siendo establecida en aproximadamente 650 años 14C AP (ca. cal. 600 años AP). Dadas las condiciones de depósitos arenosos en esta región, la expectativa general es que los sitios a cielo abierto no estuvieran enterrados y/o que presenten una muy baja resolución producto de la mezcla estratigráfica generada por la dinámica natural de los mismos. Los resultados de este trabajo confirman que el depósito no es un sitio somero, generado por migraciones de materiales superficiales o incluidos en horizonte A de un suelo (Zárate et al. 2000/2002). En términos generales, la diacronía es coherente con la estratigrafía, aunque también se registraron distribuciones verticales internas a cada conjunto, las cuales son concordantes con las expectativas temporales. Los restos cerámicos se restringen a los niveles superiores, mientras que la estructura del registro lítico no es homogénea, sino que es variable entre conjuntos diacrónicos. Esta situación no significa que todo depósito a cielo abierto 
se comporte del mismo modo, ni que cada uno de los conjuntos temporales presente alta resolución temporal. El ensamble cerámico de fragmentos de una misma pieza distanciados hasta $30 \mathrm{~cm}$, confirma la existencia de migración vertical de materiales. Por estas evidencias, sostenemos la segregación temporal de dos conjuntos, pero cada uno con una baja resolución temporal.

La ocupación definida entre 1900 y 650 años 14C $A P$, denominada conjunto $B$, muestra un registro significativamente menos denso, manifestado en una tasa temporal fuertemente inferior a la estimada para el conjunto A, posterior a los 650 años y estimativamente hasta unos 200 años atrás. Asimismo, el conjunto lítico presenta diferencias en los sistemas de producción. El conjunto B puede ser caracterizado como un sistema de producción secuencial donde las actividades de aprovisionamiento y uso de los líticos se dan en diferentes áreas o locus, contrario a lo documentado en el conjunto $A$, que responde a un sistema de producción terminal, el cual implica que todas las etapas de producción están representadas en el sitio. Estas diferencias permiten sostener que esta unidad más antigua fue producto de ocupaciones de estadías más breves y/o mayor movilidad que las registradas en el conjunto $A$. Los petroglifos contiguos, definidos como sitio Cor-2 se destacan por la presencia de figuras geométricas "mascariformes" (sensu Gradin 1997/1998: 14). A escala regional, estos tipos de motivos han sido datados de manera relativa como previos al 1300 años AP (siglo VIII según Gradin 1997/1998: 14), lo cual es consistente con el rango temporal definido radiocarbónicamente para el conjunto B. En escala regional, los motivos geométricos "mascariformes" se han registrado en sitios de los desiertos de Patagonia y Monte, al Sur del río Atuel y al Norte del río Colorado (Gradin 1997/1998; Lagiglia 1997; Schobinger 2002).

Los sitios JCP-1 y JCP-2 tienen repertorios similares entre sí, con una presencia mayoritaria de motivos asignables al "estilo de grecas" (Gradin 1997/1998; Menghin 1957) o "tendencia abstracta geométrico-compleja" (TAGC; Gradin 1988, 2001) producidos mediante pintura, principalmente de color rojo. A ello se suma el hecho de que en JCP-1 se registran motivos asignables al estilo "pisadas" (Gradin 1997/1998; Menghin 1957), que se produjeron mediante técnicas de grabado y, también, técnica mixta (grabado repintado, muy poco frecuente en todo Patagonia). Estos últimos, no están presentes en JCP-2, donde tampoco hay uso de las técnicas de grabado: esto enfatiza el vínculo entre técnicas y morfologías de los motivos artísticos (geométricos=pintados; pisadas=grabados). La presencia de motivos asignables a la TAGC y al estilo de pisadas refuerza la importante distribución espacial de ambas tendencias artísticas a lo largo de la Patagonia, incluyendo el Sur de Mendoza (Gradin 1997-98; Schobinger 2002). A su vez, ello permite inferir la existencia de circulación y/o interacción de poblaciones al Norte y al Sur del río Colorado. La presencia de motivos de la TAGC, datada de manera relativa en sitios de Patagonia ca. 1100 y 700 años AP (Crivelli Montero 2006; Fernández 2006; Gradin 1997/1998) es consistente con la fecha estimada como el límite temporal del conjunto B y conjunto $A$. Los motivos geométricos complejos pintados se ubican tanto en la zona de piedemonte como en la planicie, al Norte y al Sur del río Atuel (Gradin 1997/1998; Lagiglia 1977; Schobinger 2002).

Al comparar el arte rupestre de JCP-1 y JCP-2 con el de Cor-2 resulta notorio que comparten un total de siete grupos tipológicos de sus respectivos repertorios de motivos: figuras geométricas complejas curvilíneas, líneas simples rectilíneas, figuras geométricas complejas rectilíneas, líneas complejas rectilíneas, líneas simples curvilíneas, figuras geométricas simples rectilíneas y figuras geométricas complejas mixtas. Dentro de estos grupos tipológicos, la variedad de diseños de los tipos de motivos es amplia, por lo cual inicialmente no puede inferirse la existencia de circulación de información inter-sitio a nivel de la especificidad morfológica de cada diseño. A ello se suma que, llamativamente, estos grupos de motivos están enteramente pintados en JCP-1 y JCP2 , mientras que se los registra exclusivamente grabados en Cor-2. Sin embargo, se observa la presencia de motivos de pisadas (tridígitos) tanto en JCP-1 (grabados y grabados repintados) como en Cor-2 (grabados): esto permite sugerir que, por lo menos, algunos motivos rupestres habrían circulado a escala de la localidad.

La Figura 7 señala la suma de densidad de probabilidades ("SPD" en inglés; Crema et al. 2017) comparativa entre los desiertos de Monte y Patagonia del sector Nordpatagónico (34 a $37^{\circ} \mathrm{LS}$ ) basados en datos radiocarbónicos $(n=314)$ del Noroeste patagónico (Gil et al. 2014; Pérez et al. 2016). Ambos desiertos tienen inicios de ocupaciones hacia fines del Pleistoceno tardío, destacándose poca variación hasta el Holoceno tardío. En Patagonia, se observan incrementos iniciales mayores a los esperados, en fechas de aproximadamente 3300 y 2600 años cal. AP asociados a casi nula evidencia de ocupación en Monte (Figura 7). Tanto en Monte como Patagonia, se registra un fuerte incremento en ca. 2300 cal AP, aunque dentro de las tendencias esperadas por el modelo (Crema et al. 2017). En los últimos 2000 años, se definen dos momentos particularmente significativos para la cronología de El Corcovo: por una parte desvíos significativos positivos, "pulsos", en el Monte para fechas cal. 1926 1805 años cal. AP y 609 549 años cal. AP que son coetáneos con cronologías de desvíos negativos significativos en Patagonia. Estos pulsos positivos del Monte indican un incremento mayor al esperado por el modelo. Ambos pulsos son contemporáneos a las dos fechas numéricas disponibles para las ocupaciones de Cor-1 (1816 a 1734 años cal. AP y 625 a 550 años cal. AP). Es decir, tanto la fecha considerada como 
inicio de las ocupaciones en Corcovo (Cor-1) como la que indica un cambio en la ocupación del sitio son contemporáneas con los dos pulsos significativamente positivos del Monte y significativamente negativo en Patagonia. Esto implicaría que tanto el inicio de la ocupación en Cor-1 como el cambio notado ca. 600 años $A P$, se dan en fechas donde regionalmente se notan variaciones poblacionales significativas. Estos dos eventos se asocian con un incremento mayor de lo esperable para esas fechas en la intensidad de ocupación y/o demografía humana del Monte y una caída también mayor a lo esperada para Patagonia. Entre ambos pulsos positivos del Monte, se registran dos que se mantienen en el rango de lo esperado por el modelo, sin superar el valor del primero e inferior al segundo, en ca. 1300 y entre 1000 y 800 años cal AP.

La fecha inicial de Cor-1 confirma la ocupación de ambientes marginales, en este caso áridos y alejados de fuentes de agua estables hacia el final del Holoceno tardío, desde unos ca. 2000 años AP (Gil 2006; Neme et al. 2005) y es coincidente con el primer pulso. El registro asociado a esta cronología señalaría una ocupación efectiva inicial de Payunia, aunque con un registro de baja intensidad, en este sector del Monte. Esta ocupación inicial es concordante con la estructura arqueológica macro regional en la que se postula el inicio de ocupaciones efectivas para Nevado-Payunia, desde ca. 2000 años (Gil 2006). Neme (2007) y Gil (2006) han explicado esta cronología más tardía en Nevado-Payunia (como también en Alta Cordillera) respecto a valles intermontanos (desierto Patagónico), como resultado de un crecimiento poblacional regional y su consecuente ocupación de ambientes previamente ignorados. Es una de las formas en que las poblaciones humanas habrían resuelto, mediante la intensificación, desbalances iniciales entre población y recursos (Neme 2007; Neme et al. 2005). Adicionalmente, el registro del conjunto B de Cor-1 señala que las poblaciones contaban con un fuerte conocimiento de los recursos locales tanto faunísticos como líticos. La posible asociación de los grabados de Cor- 2 a esta fecha también indicaría la incorporación de este sector a los rangos de acción humana.

En el desierto del Monte en el noroeste de Patagonia, luego de ca. 1900 cal. AP. se destaca el segundo pulso significativamente mayor con la fecha de ca. 600-550 años cal AP, el cual es más breve y más fuerte que los previos. Posteriormente, la tendencia declina a valores semejantes a los pre- 600-550 años AP (Figura 7). Este último pulso (600-550 años cal. AP), notablemente más

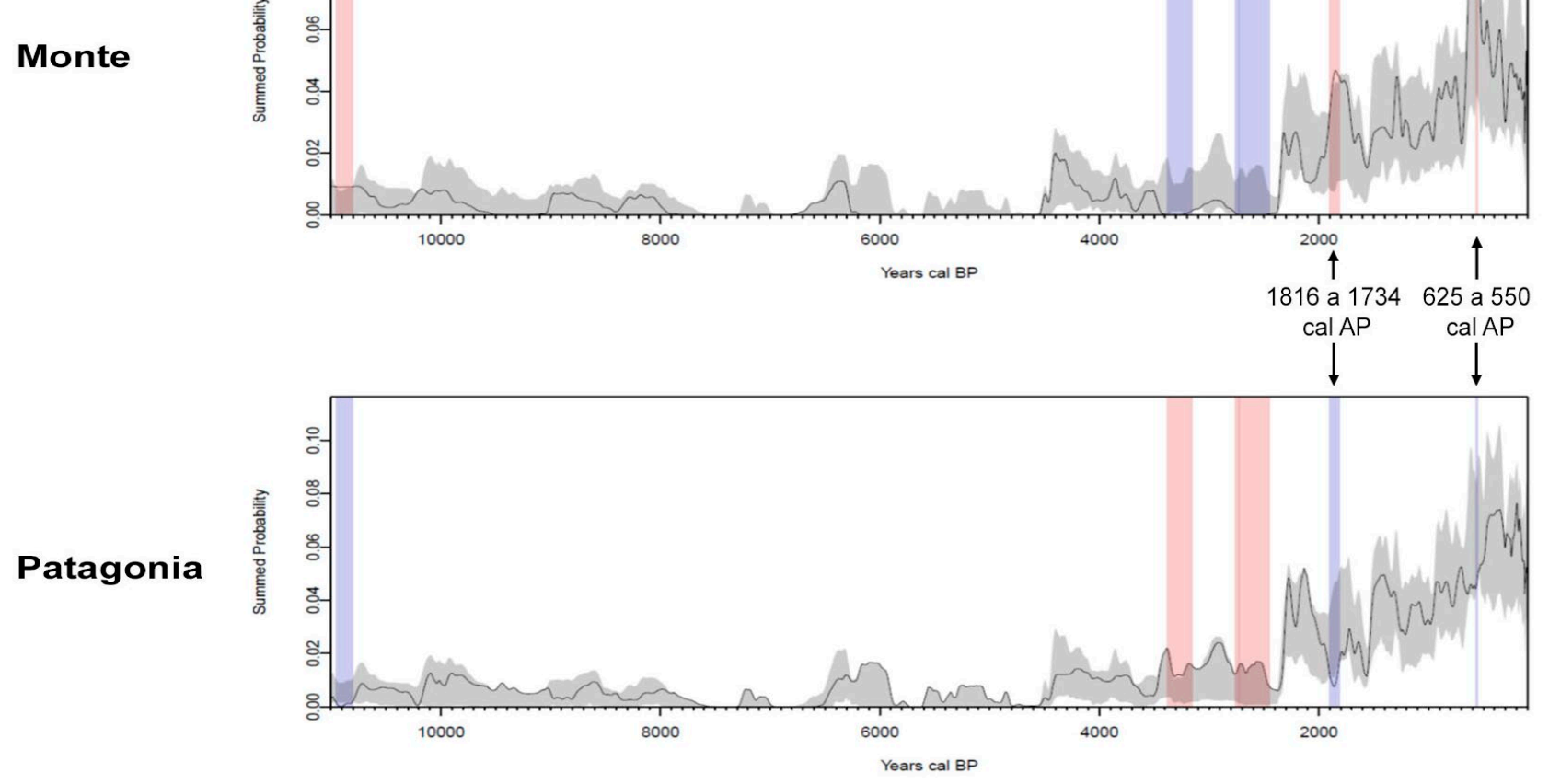

Figura 7. Suma de probabilidad de fechados radiocarbónicos comparativos entre Monte y Patagonia (34 a $37^{\circ}$ LS). Análisis realizado con RCarbon (Bevan y Crema 2018). Áreas rojas, exceden significativamente lo esperado por la comparación, mientras que la gris disminuyen más de lo esperado.

Figure 7. Sum of probability of comparative radiocarbonic dates between Monte and Patagonia (34 a $37^{\circ}$ LS). Analysis performed with RCarbon (Bevan y Crema 2018). Red areas are those where regional population exceeds the expectations of the null, blue areas are those where population is beneath expectations. 
fuerte, podría explicar, al menos parcialmente, el cambio en el patrón de ocupación respecto al pulso asociado al conjunto B en El Corcovo. También sería contemporáneo al movimiento poblacional Nordpatagónico propuesto para estas fechas por diversos autores (Barrientos y Pérez 2004; Beguelín et al. 2006). El contexto del conjunto $A$, asociado con este pulso significativamente más alto a lo esperado, se vincula con un uso (o al menos un mayor uso) de la tecnología cerámica, posiblemente con la producción de pinturas geométricas en JCP-1 y JCP-2 (vinculables al estilo de grecas o TAGC) y con una ocupación más intensa, con estadías más prolongadas y/o re-ocupaciones más frecuentes del sitio Cor-1. La subsistencia durante este momento fue basada en el mismo grupo de recursos que en el conjunto previo, confirmando la importancia para toda la ocupación de Cor-1 de recursos faunísticos pequeños de alto ranking (Corbat et al. 2016), principalmente armadillos y huevos de reidos.

La contemporaneidad de estas fechas (inicio de ocupación y cambio depositacional) de Cor-1, con los pulsos regionales de incremento significativo en Monte y decrecimiento significativo en Patagonia, podría estar señalando una complementariedad entre los dos desiertos, al menos, en las fechas mencionadas. Entonces, los pulsos de ocupación del Monte durante los últimos 2000 años podrían responder, al menos en estos casos mencionados, a momentos de incremento sostenido de la población probablemente por reposicionamiento espacial asociado a disminuciones poblacionales significativas en Patagonia.

\section{Conclusiones}

Los estudios arqueológicos en el sector Monte del Noroeste patagónico señalan trayectorias de cambio previamente inesperados en la historia humana regional (Aguerre 2002; Berón y Curtoni 1998; Gordón et al. 2017, 2018; Gradin 1984; Rindel et al. 2018; Tripaldi et al. 2017). Si bien estudios previos resaltaron la dificultad de encontrar depósitos enterrados a cielo abierto del Monte (Aguerre 2002; Austral 1971), los trabajos en El Corcovo muestran que tal posibilidad existe $y$, adicionalmente, informan de una alta concentración y diversidad de arte rupestre potencialmente asociada a ocupaciones humanas de, al menos, 1900 años AP, corroborando lo propuesto por Rindel et al. (2018). Asimismo, el registro de Cor-1 define un cambio en la estructura arqueológica en torno a los 600 años $A P$, momento desde el que se nota una ocupación más extensa y/o reocupaciones más frecuentes. Este cambio se asoció con variaciones en la tecnología ya que previamente no hay registro cerámico (por ausencia de uso o por una estrategia de alta movilidad y/o poca estadía que disminuye su visibilidad arqueológica). También el sistema de producción lítica cambia desde un foco secuencial, en las ocupaciones previas del conjunto $B$, hacia una producción terminal, desde el rango de distancia local. Junto a estas variaciones, se postula la asociación diacrónica del arte rupestre, basado en cronologías estilísticas regionales. La baja depositación de material fuera de Cor-1, Cor-2, JCP-1 y JCP-2, que se asocian fuertemente a las aguadas, confirman lo notado en las prospecciones de Bajo Añelo, Auca Mahuida y borde Sur del Alto valle del Colorado por Rindel et al. (2018).

El Corcovo confirma la ocupación efectiva tardía de La Payunia-Nevado (empleando las unidades de Neme y Gil 2005) y este sector del Monte. También, las dos fechas obtenidas muestran una asociación de estos cambios con procesos demográficos regionales del sur de Mendoza, corroborándose el inicio de ocupación de El Corcovo en un incremento significativo, aquí llamado "pulso", de la población humana en Monte, mientras que en Patagonia declinaría. Los cambios en El Corcovo a partir de 600 años AP, se vinculan regionalmente al mayor incremento poblacional del Noroeste patagónico y específicamente del Monte. Dicho incremento se dio entre 600 y 550 años cal. AP, fecha desde la cual cayó fuertemente la señal humana.

San Rafael, 30 de enero 2019

\section{Agradecimientos}

Agradecemos a la Dirección de Patrimonio CulturalGobierno de Mendoza (Lic. Elina Albarrán) a la empresa Pluspetrol, Yacimiento Corcovo (Ings. Mario Magallanes y Gastón Vázquez) y a Bios Consultores (Ing. Nancy Barreras) por el apoyo en los trabajos de campo y la receptividad a las sugerencias y recomendaciones que surgieron como parte de este trabajo. La investigación se enmarca en el PICT 2016-2667. Particularmente, agradecemos a la Familia Díaz por la información, las enseñanzas, y no menos importante, el agua convidada. Agradecemos también a los dos evaluadores quienes realizaron comentarios y sugerencias que ayudaron a mejorar sustancialmente una versión previa.

\section{Bibliografía}

Aguerre, A.M. y A.H. Tapia. 2002. Entre médanos y caldenes de la pampa seca: arqueología, historia, lengua y topónimos. Facultad de Filosofía y Letras, Universidad de Buenos Aires.

Aschero, C. 1975. Ensayo para una clasificación morfológica de artefactos líticos aplicados a estudios tipológicos comparativos. Informe a CONICET, Ms.

Aschero, C. 1983. Ensayo para una clasificación morfológica de artefactos líticos aplicada a estudios tecnológicos comparativos. Apéndice A-C. Revisión 1983, Cátedra de Ergología y Tecnología. Facultad de Filosofía y Letras, Universidad de Buenos Aires, Buenos Aires. Ms.

Aschero, C. y S. Hocsman. 2004. Revisando cuestiones 
tipológicas en torno a la clasificación de artefactos bifaciales. En: A. Acosta, D. Loponte y M. Ramos (Eds.), Temas de Arqueología, Análisis Lítico, pp. 7-26. Sociedad Argentina de Antropología, Secretaría de Cultura de la Nación, Instituto Nacional de Antropología y Pensamiento Latinoamericano, Universidad Nacional de Luján, Buenos Aires.

Austral, A. 1971. El yacimiento arqueológico Vallejo en el N.O. de la provincia de La Pampa. Contribución a la sistematización de la prehistoria y arqueología de la región pampeana. Relaciones de la Sociedad Argentina de Antropología V (2): 49-70.

Barberena R. 2015. Cueva Huenul 1 Archaeological Site (Northwestern Patagonia, Argentina): Initial Colonization and mid-Holocene Demographic Retraction. Latin American Antiquity 26: 304- 318.

Barberena, R., L. Prates y M. E. De Porras. 2015. The human occupation of northwestern Patagonia (Argentina): Paleoecological and chronological trends. Quaternary International 356: 111-126.

Barberena, R., A. Zangrando; A. Gil, G. Martinez, G. Politis, L. Borrero, G. Neme. 2009. Guanaco (Lama guanicoe) isotopic ecology in southern South America: spatial and temporal tendencies, and archaeological implications. Journal of Archaeological Science. 36: 2666-2675.

Barrientos, G. y S.I. Pérez. 2004. La expansión y dispersión de poblaciones del norte de Patagonia durante el Holoceno tardío: evidencia arqueológica y modelo explicativo. En: M.T. Civalero, P. Fernández y A.G. Guraieb (Eds.), Contra viento y marea. Arqueología de Patagonia, pp. 179-195, Instituto Nacional de Antropología y Pensamiento Latinoamericano, Sociedad Argentina de Antropología, Buenos Aires.

Beguelín, M., V. Bernal, M. del Papa, P. Novellino y G. Barrientos. 2006. El poblamiento humano tardío del sur de Mendoza y su relación con el norte de Patagonia: una discusión bioarqueológica. Anales de Arqueología y Etnología 61: 5-25.

Berón, M. y R. Curtoni. 1998. Investigaciones arqueológicas en la Subregión Pampa Seca, cuenca del río Curacó, Pcia. de La Pampa. Intersecciones en Antropología 2: 5-30.

Bevan, A. y Crema, E.R. 2018. rcarbon v1.2.0: Methods for calibrating and analyzing radiocarbon dates URL: https://CRAN.R-project.org/package=rcarbon

Borrero, L.A. 1989. Spatial heterogeneity in FuegoPatagonia. En: S. Shennan (Ed.) Archaeological Approaches to Cultural Identity, pp. 258-265. Unwin Hyman.
Borrero, L.A. 2009. The Elusive Evidence: The Archaeological Record of the South American Extinct Megafauna. En: G. Haynes (Ed.), American Megafaunal Extinctions at the End of the Pleistocene, pp. 145-68. Springer Science, Dordrecht.

Corbat, M., A. Gil y A.F. Zangrando. 2016. Ranking de recursos y el rol de los peces en las dietas humanas del centro occidente argentino. En: M.C. Salemme et al. (Comps.), Libro de Resúmenes del IV Congreso Nacional de Zooarqueología Argentina, pp. 92. Universidad Nacional de Tierra del Fuego, Ushuaia.

Cordero, R., S. Lanzelotti, y H. Panarello. 2002. INGEIS, Radiocarbon Laboratory Dates IV. Radiocarbon. 44 (1): 181-193.

Crema, E.R., A. Bevan y S. Shennan. 2017. Spatiotemporal approaches to archaeological radiocarbon dates. Journal of Archaeological Science 87: 1-9. doi:10.1016/j.jas.2017.09.007

Crivelli Montero, E.A. 2006. Frecuencia de creación de sitios de arte rupestre en la cuenca superior y media del río Limay (noroeste patagónico). En: D. Fiore y M.M. Podestá (Eds.), Tramas en la Piedra. Producción y usos del arte rupestre, pp. 63-74. Sociedad Argentina de Antropología, Asociación de Amigos del Instituto Nacional de Antropología, World Archaelogycal Congress, Buenos Aires.

Durán, V. y R. Mikkan 2009. Impacto Del volcanismo holocénico sobre el poblamiento humano del sur de Mendoza (Argentina). Intersecciones en Antropología 10: 295-310.

Durán, V., D. Winocur, C.R. Stern, R. Garvey, R. Barberena, J.L. Peña Monné y A. Benítez. 2016. Impacto del volcanismo y glaciarismo holocénicos en el poblamiento humano de la cordillera sur de Mendoza (Argentina): una perspectiva geoarqueológica. Intersecciones en Antropología 4: 33-46.

Ericson, J.E. 1984. Toward the analysis of lithic production system. En: J.E. Ericson y B. Purdy (Eds.), Prehistoric Quarries and Lithic Production, pp. 1-9. Cambridge University Press, Cambridge.

García, A. 2010. Human Occupation during the MidHolocene in Western Argentina: A Comment on Neme and Gil. Current Anthropology 51 (3): 415-416. http:// www.jstor.org/stable/10.1086/652643

Fernández, M.M. 2006. Cronología del Estilo de Grecas en la cuenca superior y media del Río Limay. En: D. Fiore y M.M. Podestá (Eds.), Tramas en la Piedra. Producción y usos del arte rupestre, pp. 75-83. Sociedad Argentina de Antropología, Asociación de Amigos del Instituto 
Nacional de Antropología, World Archaelogycal Congress, Buenos Aires.

Fernández, M.V., R. Barberena, A.A. Rughini, M. Giesso, V. Cortegoso, V. Durán, G. Romero Villanueva, K. Borrazzo, G. Lucero, R. Garvey, B. L. Macdonald y M.D. Glascock. 2017. Obsidian geochemistry, geoarchaeology, and lithic technology in northwestern Patagonia (Argentina). Journal of Archaeological Science: Reports 13: 372-381.

Gil, A. 2006. Arqueología de la Payunia. BAR, International Series 1477, Oxford.

Gil, A. y G. Neme. 2006. Distribuciones arqueológicas superficiales en Payunia-Llancanelo. Anales de Arqueología y Etnología 61: 163-184.

Gil, A., M. Zárate y G. Neme. 2005. Mid-Holocene Paleoenvironments and the archaeological record of southern Mendoza, Argentina. Quaternary International 132: 81-94.

Gil, A., R. Villalba, A. Ugan, V. Cortegoso, G. Neme, C. Michieli, P. Novellino y V. Durán. 2014. Isotopic evidence on human bone for declining maize consumption during the Little Ice Age in central western Argentina. Journal of Archaeological Science 49: 113-227.

Gil, A., A. Ugan, C. Otaola, G. Neme, M. Giardina y L. Menendez. 2016. Variation in camelid d13C and d15N values in relation to geography and climate: Holocene patterns and archaeological implications in central western Argentina. Journal of Archaeological Science 66: 7-20.

Gould, R.A. 1991. Arid-land foraging as seen from Australia: adaptative models and behavioral realities. Oceanía 62: 12-33.

Gordón, F., R. Barberena y V. Bernal (Eds.). 2017. El Poblamiento Humano del Norte del Neuquén. Estado Actual del Conocimiento y Perspectivas. Aspha, Buenos Aires.

Gordón, F., S.I. Perez, A. Hajduk, M. Lezcano y V. Bernal. 2018. Dietary patterns in human populations from northwest Patagonia during Holocene: an approach using Binford's frames of reference and Bayesian isotope mixing models. Archaeological and Anthropological Sciences 10: 1347-1358. https://doi.org/10.1007/ s12520-016-0459-0.

Gradin, C. J. 1984. Investigaciones arqueológicas en Casa de Piedra. Dirección General de Cultura, Provincia de la Paampa, Ente Ejecutivo Presa Embalse Casa de Piedra, Buenos Aires.

Gradin, C. J. 1988. Caracterización de las tendencias estilísticas del arte rupestre de la Patagonia (provincias de Río Negro, Chubut y Santa Cruz, República Argentina). Nuevos estudios del arte rupestre argentino. Contribuciones al estudio del arte sudamericano. Boletín SIARB 2: 54-67.

Gradin, C.J. 1997-1998. El arte rupestre del sur mendocino entre los siglos VIII y XV de la era ¿Un área de conflicto o de convivencia? Relaciones de la Sociedad Argentina de Antropología XXII-XXIII: 7-23.

Gradin, C. J. 2001. El arte rupestre de los cazadores de guanaco de la Patagonia. En: E. Berberián y A. Nielsen (Eds.), Historia Argentina Prehispánica, pp. 839-874. Brujas, Córdoba.

Harman, J. 2008. Using Decorrelation Stretch to Enhance Rock Art Images. http://www.dstretch.com/ AlgorithmDescription.html (Acceso junio de 2018).

Ingbar, E. 1994. Lithic Material Selection and Technological Organization. En: P. Carr (Ed.), The organization of North American prehistoric chipped stone Technologies, pp. 45-56. International Monographs in Prehistory, Archaeological Series 7, Michigan.

Ingbar, E., M. Larson y B. Bradley. 1989. A Nontypological Approach to Debitage Análisis, En: D.S. Amick y R.P. Mauldin (Eds.), Experiments in Lithic Technology, pp. 6799, BAR International Series 528, Oxford.

Jochim, M. 1981. Strategies for Survival. Cultural Behavior in an Ecological Context. Academic Press, New York.

Lagiglia, H. 1977. Arqueología y ambiente natural de los valles del Atuel y del Diamante. Tesis Doctoral inédita, Facultad de Ciencias Naturales y Museo, Universidad Nacional de La Plata, La Plata.

Lagiglia, H. 1997. Arqueología de CazadoresRecolectores Cordilleranos de Altura. Ediciones Ciencia y Arte, Instituto de Ciencias Naturales de San Rafael, Museo Municipal de Historia Natural, San Rafael.

Martínez, G., M. Gutiérrez, P. Messineo, C. Kaufmann y D.J. Rafuse. 2016. Subsistence strategies in Argentina during the Late Pleistocene and Early Holocene. Quaternary Science Reviews 144: 51-65.

Méndez, C., A. Gil, G. Neme, A. Nuevo Delaunay, V. Cortegoso, C. Huidobro, V. Durán y A. Maldonado. 2014. Mid Holocene radiocarbon ages in the subtropical Andes ( 29e35 S), climate change and implications for human space organization. Quaternary International 356: 1526. http://dx.doi.org/10.1016/j.quaint.2014.06.059

Menghin, O. 1957. Los estilos del arte rupestre de Patagonia. Acta Prehistórica 1: 57-87. 
Neme, G. 2002. Arqueología del alto valle del río Atuel: modelos, problemas y perspectivas en el estudio arqueológico de las regiones de altura del sur de Mendoza. En: A. Gil y G. Neme (Eds.), Entre Montañas y Desiertos: Arqueología del Sur de Mendoza, pp. 65-84. Sociedad Argentina de Antropología, Buenos Aires.

Neme, G. 2007. Cazadores Recolectores de Altura en los Andes Meridionales. BAR, International Series 1591, Oxford.

Neme, G. y A. Gil. 2002. La explotación faunística y la frecuencia de partes esqueletales en el registro arqueológico del sur Mendocino. En: A. Gil y G. Neme (Eds.), Entre montañas y desiertos: arqueología del sur de Mendoza, pp. 141-155. Sociedad Argentina de Antropología, Buenos Aires.

Neme, G. y A. Gil. 2008. Biogeografía Humana en los Andes Meridionales: Tendencias Arqueológicas en el sur de Mendoza. Chungara 40 (1): 5-158.

Neme, G. y A. Gil. 2009. Human occupation and increasing Mid-Holocene Aridity. Current Anthropology 50 (1): 149-163.

Neme, G. y A. Gil. 2012. El Registro Arqueológico del sur de Mendoza en Perspectiva Biogeográfica. En: G. Neme y A. Gil (Comps.), Paleoecología Humana en el Sur de Mendoza: Perspectivas Arqueológicas, pp. 254279. Sociedad Argentina de Antropología, Buenos Aires.

Neme, G., A. Gil y V. Durán. 2005. Late Holocene in Northwestern Patagonia: radiocarbon pattern and human occupation. Before Farming 2: 197-214.

Neme, G., A. Gil, R. Garvey, C. Llano, A. Zangrando, F. Franchetti, C. de Francesco, y C.T. Micheli. 2011. El registro arqueológico de la gruta de El Manzano y sus implicancias para la arqueología de Nord Patagonia. Magallania 39 (2): 243-265.

Orton, C., P. Tyers y A. Vince [1993] 1997. La cerámica en Arqueología. Ed. Crítica, Barcelona.

Pérez, S.I., V. Bernal y P.N. González. 2016. Past population dynamics in Northwest Patagonia: an estimation using molecular and radiocarbon data. Journal of Archaeological Science 65: 154-160.

Pompei, M.P. 2018. Organización de la tecnología lítica en la cuenca del río Atuel (sur de la provincia de Mendoza) durante el Holoceno tardío. Tesis doctoral inédita, Facultad de Ciencias Naturales y Museo, Universidad Nacional de La Plata, La Plata.

Prates, L., G. Politis y J. Steele. 2013. Radiocarbon Chronology of the Early Human Occupation of Argentina.
Quaternary International 301: 104-122. https://doi. org/10.1016/j.quaint.2013.03.011

Rick, J. 1987. Dates as Data: An Examination of the Peruvian Pre-Ceramic Radiocarbon Record. American Antiquity 52: 55-73.

Rindel, D., S.I. Perez, G. Romero Villanueva, D. Gobbo y A. Feely. 2018. Investigaciones arqueológicas en el noreste de Neuquén: evidencias materiales y tendencias distribucionales preliminares. Intersecciones en Antropología 19: 99-110.

Rughini, A.A. 2016. Tecnología lítica del noreste de Neuquén. Análisis del sitio Cueva Huenul 1 (Departamento Pehuenches, localidad Barrancas, Provincia de Neuquén, Argentina. Tesina de Licenciatura inédita, Facultad de Filosofía y Letras, Universidad de Buenos Aires, Buenos Aires.

Rye, O. 1988. Manual on Archaeology 4. Pottery Technology. Principles and Reconstruction. Australian National University, Taraxacum, Washington.

Salgán, M.L. 2013. Organización tecnológica y biogeografía humana en La Payunia, sur de la Provincia de Mendoza. Tesis Doctoral inédita, Facultad de Ciencias Naturales y Museo, Universidad Nacional de La Plata, La Plata.

Salgán, M.L. 2015. Disponibilidad, estrategias de aprovisionamiento y uso de recursos líticos en La Payunia, sur de Mendoza. Revista del Museo de Antropología 8 (2): 119-132.

Shennan, S., S.S. Downey, A. Timpson, K. Edinborough, S. Colledge, T. Kerig, K. Manning y M. G. Thomas. 2013. Regional population collapse followed initial agriculture booms in mid-Holocene Europe. Nature Communications 4: 2486. https://doi.org/10.1038/ncomms3486

Schobinger, J. 2002. Arte rupestre del departamento de Malargüe. En: A. Gil y G. Neme (Eds.), Entre Montañas y Desiertos: Arqueología del Sur de Mendoza, pp. 103118. Sociedad Argentina de Antropología, Buenos Aires.

Steele, J. y G. Politis. 2009. AMS 14C dating of early human occupation of southern South America. Journal of Archaeological Science 36 (2): 419-429.

Sugrañes, N. 2017a La tecnología cerámica y su relación con las estrategias de subsistencia y movilidad de poblaciones humanas en la cuenca del Atuel (sur de Mendoza), durante el Holoceno tardío. Tesis doctoral inédita, Facultad de Ciencias Sociales, Universidad del Centro de la Provincia de Buenos Aires, Olavarría.

Sugrañes, N. 2017b. Análisis de Formas sobre Vasijas 
Enteras de Museos del sur de Mendoza. Revista del Museo de Antropología 10 (1): 37-42.

Sugrañes, N. y F. Franchetti. 2012. Antecedentes, problemas y perspectivas del análisis cerámico en el sur de Mendoza. Una puesta al día. En: G. Neme y A. Gil (Eds.). Paleoecología Humana en el Sur de Mendoza: Perspectivas Arqueológicas, pp. 229-253. Sociedad de Antropología Argentina, Buenos Aires.

Tripaldi, A., M. Zárate, G. Neme, A. Gil, M.A. Giardina y M.L. Salgán. 2017. Archaeological site formation processes in northwestern Patagonia, Mendoza
Province, Argentina. Geoarchaeology International Journal 32 (6): 605-621.

Yellen, J. 1977. Long term hunter-gatherer adaptation to desert environments: a biogeographical perspective. World Archaeology 8: 262-274.

Zárate, M., M. González, N. Flegenheimer y C. Bayón. 2000-2002. Sitios arqueológicos someros: el concepto de sitio en estratigrafía y sitio de superficie. Cuadernos del Instituto Nacional de Antropología y Pensamiento Latinoamericano 19: 635-653. 